\title{
DEPENDENT LINDEBERG CENTRAL LIMIT THEOREM AND SOME APPLICATIONS
}

\author{
Jean-Marc Bardet ${ }^{1}$, Paul Doukhan ${ }^{1,2}$, Gabriel Lang $^{3}$ and Nicolas Ragache ${ }^{2}$
}

\begin{abstract}
In this paper, a very useful lemma (in two versions) is proved: it simplifies notably the essential step to establish a Lindeberg central limit theorem for dependent processes. Then, applying this lemma to weakly dependent processes introduced in Doukhan and Louhichi (1999), a new central limit theorem is obtained for sample mean or kernel density estimator. Moreover, by using the subsampling, extensions under weaker assumptions of these central limit theorems are provided. All the usual causal or non causal time series: Gaussian, associated, linear, $\operatorname{ARCH}(\infty)$, bilinear, Volterra processes, ..., enter this frame.
\end{abstract}

Mathematics Subject Classification. 60F05, 62G07, 62M10, 62 G09.

Received June 15, 2007. Revised June 27, 2007.

\section{INTRODUCTION}

This paper adresses the problem of the central limit theorem (CLT) for weakly dependent sequences with the point of view of the classical Lindeberg method (see Petrov [15], for references Rio [17]). For establishing a CLT for a sequence $\left(S_{n}\right)_{n \in \mathbb{N}^{*}}$ of random vectors (r.v.), a convenient and efficient method (so-called the "Lindeberg's method" in the sequel) consists on proving that for all functions $f$ with bounded and continuous partial derivatives up to order 3 ,

$$
\left|\mathbb{E}\left(f\left(S_{n}\right)-f(N)\right)\right| \underset{n \rightarrow \infty}{\longrightarrow} 0,
$$

with $N$ a Gaussian random variable not depending on $n$. Assume that $S_{n}=X_{1}+\cdots+X_{n}$ where $\left(X_{k}\right)_{k \in \mathbb{N} *}$ is a zero-mean sequence with $(2+\delta)$-order finite moments for some $\delta>0$, and consider $\left(Y_{k}\right)_{k \in \mathbb{N}^{*}}$ a sequence of independent zero mean Gaussian r.v. such that the variance of $Y_{k}$ and $X_{k}$ are the same. In order to obtain (1), we first show that this convergence is satisfied when the sum of two terms converges to zero. The first term is the sum of the $(2+\delta)$-order moments of $\left(X_{k}\right)_{1 \leq k \leq n}$. The second term is a sum of covariances between functions of $\left(X_{k}\right)_{1 \leq k \leq n}$ and $\left(Y_{k}\right)_{1 \leq k \leq n}$ and reflects all the dependence structure. Three cases are thus detailed in three different lemmata: first, the case of independent r.v.s (here, the second term vanishes), then the dependent case with general functions $f$, and finally the dependent case with characteristic functions that yields a very simple expression.

\footnotetext{
Keywords and phrases. Central limit theorem, Lindeberg method, weak dependence, kernel density estimation, subsampling.

1 Samos-Matisse-CES, Université Panthéon-Sorbonne, 90 rue de Tolbiac, 75013 Paris, France.

2 LS-CREST, Timbre J340, 3 avenue Pierre Larousse, 92240 Malakoff, France.

3 AgroParisTech, UMR MIA 518 (AgroParisTech-INRA), 75005 Paris, France.
} 
For applications of those lemmata, the class of weakly dependent processes, introduced by Doukhan and Louhichi [9], is selected here. Roughly speaking, a process $X=\left(X_{k}\right)_{k \in \mathbb{N}^{*}}$ is said to be a weakly dependent process if the covariance of any bounded and Lipschitz function of "past" data of $X$ by any bounded and Lipschitz function of "future" data of $X$ tends to 0 when the lag between the future and the past increases to $\infty$ (see a more precise definition below).

Why should we use such dependence structures (instead e.g. mixing)?

Two main reasons motivate this choice. Firstly, weak dependence is a very general property including certain non-mixing processes: e.g. Andrews [1] explicited the simple example of an autogressive process with Bernoulli innovations and proved that such a model is not mixing in the sense of Rosenblatt (see for instance Doukhan [6], or Rio [18], for references) while Doukhan and Louhichi [9] proved that such a process is weakly dependent. More generally, under weak conditions, all the usual causal or non causal time series are weakly dependent processes: this is the case for instance of Gaussian, associated, linear, $\operatorname{ARCH}(\infty)$, bilinear, Volterra, infinite memory processes, ... Secondly, the dependence property is obtained from the convergence to zero of covariances of the process (see above). The second term in our lemmata writes as a sum of covariances; weak dependence is therefore particularly accurate to bound this term (which is the essential step for proving the Lindeberg CLT).

Different applications of the lemmata are then presented for weakly dependent processes. First, a CLT for sample means is established in Doukhan and Wintenberger [12]; in addition to the previous lemma for characteristic function, the Bernstein block method is required (in such a case, Bulinski and Shashkin [3] and [4] used also this method; an alternative method is derived in Rio [18] Coulon-Prieur and Doukhan [5] or Neumann and Paparoditis [14]). For weakly dependent processes, a CLT for the subsample mean is derived here directly from our lemmata. By this way, the conditions required for such a theorem are weaker than those required for the CLT for the sample mean. For instance, the subsampling of a long range dependent process provides a CLT for its sample mean, which is interesting for obtaining confidence intervals or semi-parametric tests (even if a large part of the sample is not used).

Finally, an application of the Lindeberg method to the kernel density estimation is also given. By this way, the CLT is established under the same conditions as in Coulon-Prieur and Doukhan [5] for causal processes (but with a more simple and general method). Its extension to non-causal processes is also proposed here. The required conditions are of a different nature that the usual conditions under strong mixing (see Robinson [19]); but on some examples of time series (for instance, the causal linear processes) they imply the same decay rates of the coefficients. On other examples (some non-causal time series), it is very difficult to check the strong mixing property. Therefore the CLT we proved concerns a lot of new models. Moreover, a version of this CLT for subsampled kernel density estimator is given. Once again, this allows to obtain a CLT under weaker conditions. With an adapted subsampling step, the asymptotic normality of this estimator is established even in the case of long memory processes, which provides usual confidence intervals on the density or goodness-of-fit tests.

The paper is organized as follows. In Section 2, the Lindeberg method is presented. Section 3 is devoted to a presentation of weakly dependent processes. Section 4 contains different applications of the Lindeberg method for weakly dependent processes while the proofs of the different results are in the Section 5.

\section{LindeBERG MEthod}

Let $\left(X_{i}\right)_{i \in \mathbb{N}}$ be a sequence of zero mean r.v. with values in $\mathbb{R}^{d}$ (equipped with the Euclidean norm $\left\|X_{i}\right\|^{2}=$ $\left(X_{i}^{(1)}\right)^{2}+\cdots+\left(X_{i}^{(d)}\right)^{2}$ for $X_{i}=\left(X_{i}^{(1)}, \ldots, X_{i}^{(d)}\right)$. Moreover, all along this paper we will assume that $\left(X_{i}\right)_{i \in \mathbb{N}}$ satisfies, 
Assumption $H_{\delta}$ : It exists $0<\delta \leq 1$ such that $\forall i \in \mathbb{N}, \mathbb{E}\left\|X_{i}\right\|^{2+\delta}<\infty$ and $\forall k \in \mathbb{N}^{*}$, define

$$
A_{k}=\sum_{i=1}^{k} \mathbb{E}\left\|X_{i}\right\|^{2+\delta} .
$$

Let $\left(Y_{i}\right)_{i \in \mathbb{N}}$ be a sequence of zero mean independent r.v. with values in $\mathbb{R}^{d}$, independent of the sequence $\left(X_{i}\right)_{i \in \mathbb{N}}$ and such that $Y_{i} \sim \mathcal{N}_{d}\left(0, \operatorname{Cov} X_{i}\right)$ for all $i \in \mathbb{N}$. Denote by $\mathcal{C}_{b}^{3}$ the set of bounded functions $\mathbb{R}^{d} \rightarrow \mathbb{R}$ with bounded and continuous partial derivatives up to order 3. Set, for $f \in \mathcal{C}_{b}^{3}$ and $k \in \mathbb{N}^{*}$,

$$
\Delta_{k}=\left|\mathbb{E}\left(f\left(X_{1}+\cdots+X_{k}\right)-f\left(Y_{1}+\cdots+Y_{k}\right)\right)\right| .
$$

Following the dependence between vectors $X_{i}$, we now provide 3 lemmata, the first one is well known and relates to the independence case, the two others are concerned with the dependence case. Thus, first, for independent random variables, the Lindeberg lemma (see e.g. Petrov [15]) is

Lemma 1 (Lindeberg under independence). Let $\left(X_{i}\right)_{i \in \mathbb{N}}$ be a sequence of independent zero mean r.v. with values in $\mathbb{R}^{d}$ satisfying Assumption $H_{\delta}$. Then, for all $k \in \mathbb{N}^{*}$ :

$$
\Delta_{k} \leq 6 \cdot\left\|f^{(2)}\right\|_{\infty}^{1-\delta} \cdot\left\|f^{(3)}\right\|_{\infty}^{\delta} \cdot A_{k} .
$$

This lemma is restated for completeness sake but it is essentially well known.

Remark 1. Using the proof of the previous lemma, classical Lindeberg conditions may be used:

$$
\begin{gathered}
\Delta_{k} \leq 2\left\|f^{(2)}\right\|_{\infty} B_{k}(\varepsilon)+\left\|f^{(3)}\right\|_{\infty} \cdot a_{k}\left(\frac{4}{3} \varepsilon+\sqrt{B_{k}(\varepsilon)}\right) . \\
\text { where } \quad B_{k}(\varepsilon)=\sum_{i=1}^{k} \mathbb{E}\left(\left\|X_{i}\right\|^{2} \mathbb{1}_{\left\{\left\|X_{i}\right\|>\varepsilon\right\}}\right), \text { for } \varepsilon>0, k \in \mathbb{N}, \\
a_{k}=\sum_{i=1}^{k} \mathbb{E}\left(\left\|X_{i}\right\|^{2}\right)<\infty, \text { for } k \in \mathbb{N} .
\end{gathered}
$$

Moreover, these classical Lindeberg conditions derive those from Lemma 1; indeed, according to Hölder Inequality, $\mathbb{E}\left(\left\|X_{i}\right\|^{2} \mathbb{1}_{\left\{\left\|X_{i}\right\|>\varepsilon\right\}}\right) \leq\left(\mathbb{E}\left(\left\|X_{i}\right\|^{2+\delta}\right)\right)^{\frac{2}{2+\delta}}\left(\mathbb{P}\left(\left\|X_{i}\right\|>\varepsilon\right)^{\frac{\delta}{2+\delta}}\right.$, and then, using the Bienaymé-Tchebichev inequality, for all $\delta \in] 0,1\left[, B_{k}(\varepsilon) \leq \varepsilon^{-\delta} A_{k}\right.$. Consequently (4) implies,

$$
\Delta_{k} \leq 2\left\|f^{(2)}\right\|_{\infty} \varepsilon^{-\delta} A_{k}+\left\|f^{(3)}\right\|_{\infty} a_{k}\left(\frac{4}{3} \varepsilon+\varepsilon^{-\delta / 2} \sqrt{A_{k}}\right) .
$$

For the dependent case, the Lindeberg method provides the two following lemmata. First, for random vectors $Z=\left(Z^{(1)}, \ldots, Z^{(d)}\right)^{\prime}$ and $W=\left(W^{(1)}, \ldots, W^{(d)}\right)^{\prime} \in \mathbb{R}^{d}$, we will use the notations,

$$
\left\{\begin{array}{l}
\operatorname{Cov}\left(f^{(1)}(W), Z\right)=\sum_{\ell=1}^{d} \operatorname{Cov}\left(\frac{\partial f}{\partial x_{\ell}}(W), Z^{(\ell)}\right) \\
\operatorname{Cov}\left(f^{(2)}(W), Z^{2}\right)=\sum_{k=1}^{d} \sum_{\ell=1}^{d} \operatorname{Cov}\left(\frac{\partial^{2} f}{\partial x_{k} \partial x_{\ell}}(W), Z^{(k)} Z^{(\ell)}\right) .
\end{array}\right.
$$


Assume that $Z^{*}$ is independent of $W$ and admits the same distribution as $Z$; the previous expression is rewritten $\operatorname{Cov}\left(f^{(2)}(W), Z^{2}\right)=\mathbb{E} f^{(2)}(W)(Z, Z)-\mathbb{E} f^{(2)}(W)\left(Z^{*}, Z^{*}\right)$ where $f^{(2)}(W)$ is here considered as a quadratic form of $\mathbb{R}^{d}$. Then,

Lemma 2 (dependent Lindeberg lemma - I). Let $\left(X_{i}\right)_{i \in \mathbb{N}}$ be a sequence of zero mean r.v. with values in $\mathbb{R}^{d}$ satisfying Assumption $H_{\delta}$. Then,

$$
\Delta_{k} \leq T_{1}(k)+\frac{1}{2} T_{2}(k)+10 \cdot\left\|f^{(2)}\right\|_{\infty}^{1-\delta} \cdot\left\|f^{(3)}\right\|_{\infty}^{\delta} \cdot A_{k}
$$

where (empty sums are set equal to 0),

$$
T_{j}(k)=\sum_{i=1}^{k}\left|\operatorname{Cov}\left(f^{(j)}\left(X_{1}+\cdots+X_{i-1}\right), X_{i}^{j}\right)\right|, \quad j=1,2 .
$$

Characteristic functions are considered below; they provide a simpler result.

Lemma 3 (dependent Lindeberg lemma - II). Let $\left(X_{i}\right)_{i \in \mathbb{N}}$ be a sequence of zero mean r.v. with values in $\mathbb{R}^{d}$ satisfying Assumption $H_{\delta}$. For the special case of complex exponential functions $f(x)=\mathrm{e}^{i\langle t, x\rangle}$ for some $t \in \mathbb{R}^{d}$ (and where $\langle a, b\rangle$ is the scalar product in $\mathbb{R}^{d}$ ),

$$
\Delta_{k} \leq T(k)+6\|t\|^{2+\delta} A_{k}, \quad \text { where } T(k)=\sum_{j=1}^{k}\left|\operatorname{Cov}\left(\mathrm{e}^{i\left\langle t, X_{1}+\cdots+X_{j-1}\right\rangle}, \mathrm{e}^{i\left\langle t, X_{j}\right\rangle}\right)\right| .
$$

The main consequence of those three lemmata is related to the asymptotic behavior of $\sum_{i=1}^{k} X_{i}$, and provide sufficient conditions for establishing the CLT.

Theorem 1 (a Lindeberg CLT). Assume that the sequence $\left(X_{i, k}\right)_{i \in \mathbb{N}}$ satisfies Assumption $H_{\delta}$, and $A_{k} \underset{k \rightarrow \infty}{\longrightarrow} 0$, and there exists $\Sigma$ a positive matrix such that $\Sigma_{k}=\sum_{i=1}^{k} \operatorname{Cov}\left(X_{i, k}\right) \underset{k \rightarrow \infty}{\longrightarrow} \Sigma$. Moreover, assume that $T_{j}(k) \underset{k \rightarrow \infty}{\longrightarrow} 0$ for $j=1,2$ (Lem. 2) or $T(k) \underset{k \rightarrow \infty}{\longrightarrow} 0$ (Lem. 3). Then,

$$
S_{k}=\sum_{i=1}^{k} X_{i, k} \underset{k \rightarrow \infty}{\stackrel{\mathcal{D}}{\longrightarrow}} \mathcal{N}_{d}(0, \Sigma)
$$

Proof of Theorem 1. Under the assumptions of this theorem, it is clear that $\left|\mathbb{E}\left(f\left(S_{k}\right)-f\left(N_{k}\right)\right)\right| \underset{k \rightarrow \infty}{\longrightarrow} 0$ for all functions $f \in \mathcal{C}_{b}^{3}$, or for all $t \in \mathbb{R}$ and $f(x)=\mathrm{e}^{i t x}$, where $N_{k} \sim \mathcal{N}_{d}\left(0, \Sigma_{k}\right)$. According to $\mid \mathbb{E}\left(f\left(N_{k}\right)-\right.$ $f(N)) \mid \underset{k \rightarrow \infty}{\longrightarrow} 0$ where $N \sim \mathcal{N}_{d}(0, \Sigma)$, we deduce that $\left|\mathbb{E}\left(f\left(S_{k}\right)-f(N)\right)\right| \underset{k \rightarrow \infty}{\longrightarrow} 0$ and therefore $S_{k} \underset{k \rightarrow \infty}{\stackrel{\mathcal{D}}{\longrightarrow}} N$.

Following this theorem, we can remark that the condition $A(k) \underset{k \rightarrow \infty}{\longrightarrow} 0$ is the usual Lindeberg condition (with also condition $\Sigma_{k} \underset{k \rightarrow \infty}{\longrightarrow} \Sigma$, the convergence of variances), while the conditions $T_{j}(k) \underset{k \rightarrow \infty}{\longrightarrow} 0$ (for $\left.j=1,2\right)$ or $T(k) \underset{k \rightarrow \infty}{\longrightarrow} 0$ are related to the dependence structure of the sequence $\left(X_{i}\right)_{i \in \mathbb{N}}$.

\section{WEAKLY DEPENDENT PROCESSES}

We have just seen that the convergence in distribution of $S_{k}$ to a Gaussian law is obtained if $T_{1}(k)$ and $T_{2}(k)$, or $T(k)$ converge to 0 . Those terms are related to the dependence of the sequence $\left(X_{n}\right)_{n \in \mathbb{N}}$. Now, we address a 
very general class of dependent processes introduced and developped in Doukhan and Louhichi [9]. Numerous reasons justify this choice. First, this frame of dependence includes a lot of models like causal or non causal linear, bilinear, strong mixing processes or also dynamical systems. Secondly, these properties of dependence are independent of the marginal distribution of the time series, that can be as well a discrete one, Lebesgue measurable one or else. Finally, these definitions of dependence can be easily used in various statistic contexts, in particular in the case of the establishment of central limit theorems, since the previous bounds provided for $T_{1}(k), T_{2}(k)$ or $T(k)$ are written with sums of covariances (see above).

To define a such dependent processes, first, for $h:\left(\mathbb{R}^{d}\right)^{u} \rightarrow \mathbb{R}$ an arbitrary function, with $d, u \in \mathbb{N}^{*}$, denote,

Then,

$$
\operatorname{Lip} h=\sup _{\left(y_{1}, \ldots, y_{u}\right) \neq\left(x_{1}, \ldots, x_{u}\right) \in\left(\mathbb{R}^{d}\right)^{u}} \frac{\left|h\left(y_{1}, \ldots, y_{u}\right)-h\left(x_{1}, \ldots, x_{u}\right)\right|}{\left\|y_{1}-x_{1}\right\|+\cdots+\left\|y_{u}-x_{u}\right\|} .
$$

Definition 1. A process $X=\left(X_{n}\right)_{n \in \mathbb{Z}}$ with values in $\mathbb{R}^{d}$ is a so-called $(\varepsilon, \psi)$-weakly dependent process if there exist a function $\psi:\left(\mathbb{N}^{*}\right)^{2} \times\left(\mathbb{R}^{+}\right)^{2} \rightarrow \mathbb{R}^{+}$and a sequence $\left(\varepsilon_{r}\right)_{r \in \mathbb{N}}$ such that $\varepsilon_{r} \underset{r \rightarrow \infty}{\longrightarrow} 0$ satisfying,

$$
\left|\operatorname{Cov}\left(g_{1}\left(X_{i_{1}}, \ldots, X_{i_{u}}\right), g_{2}\left(X_{j_{1}}, \ldots, X_{j_{v}}\right)\right)\right| \leq \psi\left(u, v, \text { Lip }_{1}, \text { Lip }_{2}\right) \cdot \varepsilon_{r}
$$

for all $\left\{\begin{array}{l}\bullet(u, v) \in \mathbb{N}^{*} \times \mathbb{N}^{*} ; \\ \bullet\left(i_{1}, \ldots, i_{u}\right) \in \mathbb{Z}^{u} \text { and }\left(j_{1}, \ldots, j_{v}\right) \in \mathbb{Z}^{v} \text { with } i_{1} \leq \cdots \leq i_{u}<i_{u}+r \leq j_{1} \leq \cdots \leq j_{v} \\ \bullet \text { functions } g_{1}: \mathbb{R}^{u d} \rightarrow \mathbb{R} \text { and } g_{2}: \mathbb{R}^{v d} \rightarrow \mathbb{R} \text { such that } \\ \left\|g_{1}\right\|_{\infty} \leq 1,\left\|g_{2}\right\|_{\infty} \leq 1, \text { Lip } g_{1}<\infty \text { and Lip } g_{2}<\infty .\end{array}\right.$

In the sequel, two different particular cases of functions $\psi$ corresponding to two different cases of weakly dependent processes will be considered (more details can be found in Doukhan and Louhichi [9], Doukhan and Wintenberger [12]),

- If $X$ is a causal time series, i.e. there exist a sequence of functions $\left(F_{n}\right)$ and a sequence of independent random variables $\left(\xi_{k}\right)_{k \in \mathbb{Z}}$ such that $X_{n}=F_{n}\left(\xi_{n}, \xi_{n-1}, \ldots\right)$ for $n \in \mathbb{Z}$, the $\theta$-weakly dependent causal condition, for which

$$
\psi\left(u, v, \operatorname{Lip} g_{1}, \operatorname{Lip} g_{2}\right)=v \cdot \operatorname{Lip} g_{2}
$$

(in such a case, we will simply denote $\theta_{r}$ instead of $\varepsilon_{r}$ ).

- If $X$ is a non causal time series, the $\lambda$-weakly dependent condition, for which

$$
\psi\left(u, v, \operatorname{Lip} g_{1}, \operatorname{Lip} g_{2}\right)=u \cdot v \cdot \operatorname{Lip} g_{1} \cdot \operatorname{Lip} g_{2}+u \cdot \operatorname{Lip} g_{1}+v \cdot \operatorname{Lip} g_{2}
$$

(in such a case, we will simply denote $\lambda_{r}$ instead of $\varepsilon_{r}$ ).

Remark 2. It is clear that if $X$ is a $\theta$-weakly dependent process it is also a $\lambda$-weakly dependent process. The main reasons for considering a distinction between causal and non causal time series are: a/ the $\theta$-weak dependence is more easily relied to the strong mixing property; b/ some models or properties require different conditions on the convergence rate of $\left(\theta_{r}\right)$ than for $\left(\lambda_{r}\right)$.

Note first that sums of independent weakly dependent processes admit the common weak dependence property where dependence coefficients are the sums of the initial ones. We now provide a non exhaustive list of weakly dependent sequences with their weak dependence properties. In the sequel, $X=\left(X_{k}\right)_{k \in \mathbb{Z}}$ denote a weakly dependent stationary time series (the conditions of the stationarity will not be specified) and $\left(\xi_{n}\right)_{n \in \mathbb{Z}}$ is a sequence of zero mean i.i.d. random variables,

1. If $X$ is a Gaussian process and if $\lim _{i \rightarrow \infty} \operatorname{Cov}\left(X_{0}, X_{i}\right)=0$, then $X$ is a $\lambda$-weakly dependent process such that $\lambda_{r}=O\left(\sup _{i \geq r}\left|\operatorname{Cov}\left(X_{0}, X_{i}\right)\right|\right)$ (see Doukhan and Louhichi [9]). 
2. If $X$ is an associated stationary processes, then $X$ is $\lambda$-weakly dependent process such that $\lambda_{r}=$ $O\left(\sup _{i>r} \operatorname{Cov}\left(X_{0}, X_{i}\right)\right)$ (see Doukhan and Louhichi [9]).

3. If $X$ is a $A R M A(p, q)$ process or, more generally, a causal (respectively, a non causal) linear process such that $X_{k}=\sum_{j=0}^{\infty} a_{j} \xi_{k-j}$ (respectively, $\left.X_{k}=\sum_{j=-\infty}^{\infty} a_{j} \xi_{k-j}\right)$ for $k \in \mathbb{Z}$, with $a_{k}=\mathcal{O}\left(|k|^{-\mu}\right)$ with $\mu>1 / 2$, then $X$ is a $\theta$ - (respectively, $\lambda$-) weakly dependent process with $\theta_{r}=\lambda_{r}=\mathcal{O}\left(\frac{1}{r^{\mu-1 / 2}}\right)$ (see Doukhan and Lang [8], p. 3). It is also possible to deduce $\lambda$-weak dependence properties for $X$ if the innovation process is itself $\lambda$-weakly dependent (Doukhan and Wintenberger [12]).

4. If $X$ is a $\operatorname{GARCH}(p, q)$ process or, more generally, a $A R C H(\infty)$ process such that $X_{k}=\rho_{k} \cdot \xi_{k}$ with $\rho_{k}^{2}=b_{0}+\sum_{j=1}^{\infty} b_{j} X_{k-j}^{2}$ for $k \in \mathbb{Z}$ and if,

- it exists $C>0$ and $\mu \in] 0,1\left[\right.$ such that $\forall j \in \mathbb{N}, 0 \leq b_{j} \leq C \cdot \mu^{-j}$, then $X$ is a $\lambda$-weakly dependent process with $\lambda_{r}=\mathcal{O}\left(\mathrm{e}^{-c \sqrt{r}}\right)$ and $c>0$ (this is the case of $G A R C H(p, q)$ processes).

- it exists $C>0$ and $\nu>1$ such that $\forall j \in \mathbb{N}, 0 \leq b_{j} \leq C \cdot j^{-\nu}$, then $X$ is a $\lambda$-weakly dependent process with $\lambda_{r}=\mathcal{O}\left(r^{-\nu+1}\right)$ (see Doukhan [11]).

5. If $X$ is a causal bilinear process such that $X_{k}=\xi_{k}\left(a_{0}+\sum_{j=1}^{\infty} a_{j} X_{k-j}\right)+c_{0}+\sum_{j=1}^{\infty} c_{j} X_{k-j}$ for $k \in \mathbb{Z}$ (see Giraitis and Surgailis [13]) and if,

- $\left\{\exists J \in \mathbb{N}\right.$ such that $\forall j>J, a_{j}=c_{j}=0$, or,

$\left\{\begin{array}{l}\exists \mu \in] 0,1\left[\text { such that } \sum_{j}\left|c_{j}\right| \mu^{-j} \leq 1 \text { and } \forall j \in \mathbb{N}, 0 \leq a_{j} \leq \mu^{j}\right.\end{array}\right.$, then $X$ is a $\lambda$-weakly dependent process with $\lambda_{r}=\mathcal{O}\left(\mathrm{e}^{-c \sqrt{r}}\right)$, constant $c>0$;

- $\forall j \in \mathbb{N}, c_{j} \geq 0$, and $\exists \nu_{1}>2$ and $\exists \nu_{2}>0$ such that $a_{j}=\mathcal{O}\left(j^{-\nu_{1}}\right)$ and $\sum_{j} c_{j} j^{1+\nu_{2}}<\infty$, with $d=\max \left(-\left(\nu_{1}-1\right) ;-\left(\nu_{2} \delta\right)\left(\delta+\nu_{2} \log 2\right)^{-1}\right)$, then $X$ is a $\lambda$-weakly dependent process with $\lambda_{r}=\mathcal{O}\left(\left(\frac{r}{\log r}\right)^{d}\right)$ and (see Doukhan et al. [10]).

6. If $X$ is a non causal bilinear process satisfying $X_{k}=\xi_{k} \cdot\left(a_{0}+\sum_{j \in \mathbb{Z}^{*}} a_{j} X_{k-j}\right)$, for $k \in \mathbb{Z}$, where $\left\|\xi_{0}\right\|_{\infty}<$ $\infty$ (bounded random variables) and $a_{k}=\mathcal{O}\left(|k|^{-\mu}\right)$ with $\mu>1$, then $X$ is a $\lambda$-weakly dependent process with $\lambda_{r}=\mathcal{O}\left(r^{1-\mu}\right)$ (see Doukhan [11]).

7. If $X$ is a non causal finite order Volterra process such that $X_{k}=\sum_{p=1}^{\infty} Y_{k}^{(p)}$ for $k \in \mathbb{Z}$, and with $Y_{k}^{(p)}=\sum_{-\infty<j_{1}<j_{2}<\cdots<j_{p}<\infty} a_{j_{1}, \ldots, j_{p}}^{(p)} \xi_{k-j_{1}} \cdots \xi_{k-j_{p}}$ and such that it exists $p_{0} \in \mathbb{N}^{*}$ satisfying for $p>p_{0}$, $a_{j_{1}, \ldots, j_{p}}^{(p)}=0$. Then if $a_{j_{1}, \ldots, j_{p}}^{(p)}=\mathcal{O}\left(\max _{1 \leq i \leq p}\left\{\left|j_{i}\right|^{-\mu}\right\}\right)$ with $\mu>0, X$ is a $\lambda$-weakly dependent process with $\lambda_{r}=\mathcal{O}\left(\frac{1}{r^{\mu+1}}\right)$ (see Doukhan [7]). As in case 3, $\lambda$-weak dependence properties for $X$ may be proved even for $\lambda$-weakly dependent innovations.

8. If $X$ is a causal (respectively, non causal) infinite memory process such that

$$
X_{k}=F\left(X_{k-1}, X_{k-2}, \ldots ; \xi_{k}\right) \quad\left(\text { respectively, } X_{k}=F\left(X_{k-t}, t \neq 0 ; \xi_{k}\right)\right) \quad \text { for } k \in \mathbb{Z}
$$

where the function $F$ is defined on $\mathbb{R}^{\mathbb{N}}$ (respectively, $\mathbb{R}^{\mathbb{Z}}$ ) and satisfies, with $m>0,\left\|F\left(0 ; \xi_{0}\right)\right\|_{m}<\infty$ and $\left\|F\left(\left(x_{j}\right)_{j} ; \xi_{0}\right)-F\left(\left(y_{j}\right)_{j} ; \xi_{0}\right)\right\|_{m} \leq \sum_{j \neq 0} a_{j}\left|x_{j}-y_{j}\right|$, where $a=\sum_{j \neq 0} a_{j}<1$. Then $X$ is a $\theta$ (respectively, $\lambda$-) weakly dependent process with $\theta_{r}=\inf _{p \geq 1}\left\{a^{\frac{r}{p}}+\sum_{j>p} a_{j}\right\}$ (respectively, $\lambda_{r}=$ $\inf _{p \geq 1}\left\{a^{\frac{r}{p}}+\sum_{|j|>p} a_{j}\right\}$ ) (see Doukhan and Wintenberger [12]). 
9. Let $X$ be a zero-mean, second order stationary Gaussian (or linear) process. Assume that $X$ is long-range dependent: $\operatorname{Cov}\left(X_{0}, X_{k}\right)=L(k) \cdot k^{2 H-2}$ for $k \in \mathbb{N}$, with $H \in(1 / 2,1)$ (so-called Hurst parameter) and $L(\cdot)$ a slowly varying function (at $+\infty)$. Then, $X$ is a $\lambda$-weakly dependent process with $\lambda_{r}=L(r) \cdot r^{2 H-2}$. Now, different applications of Theorem 1 are considered for weakly dependent processes.

\section{Applications under weak Dependence}

\subsection{Lindeberg central limit theorem}

Doukhan and Wintenberger [12] prove a CLT using Bernstein blocks for a sequence $\left(X_{i}\right)_{i \in \mathbb{N}}$ of stationary zero mean $(2+\delta$ )-order random variables. In order to prove $T(k) \underset{k \rightarrow \infty}{\longrightarrow} 0$ (see Lem. 3), we consider two sequences $\left(p_{n}\right)_{n \in \mathbb{N}}$ and $\left(q_{n}\right)_{n \in \mathbb{N}}$ such that,

$$
\left\{\begin{array}{l}
p_{n} \underset{n \rightarrow \infty}{\longrightarrow} \infty \\
q_{n} \underset{n \rightarrow \infty}{\longrightarrow} \infty
\end{array} \text { and } p_{n}=o(n), \quad q_{n}=o\left(p_{n}\right)\right.
$$

Introduce now the number of Bernstein blocks $k_{n}=\left[\frac{n}{p_{n}+q_{n}}\right]$. It can be shown that if $p_{n} \cdot q_{n}=o(n)$,

$$
\left\|\frac{1}{\sqrt{n}} \sum_{i=1}^{n} X_{i}-\frac{1}{\sqrt{n}} \sum_{j=1}^{k_{n}} \sum_{i=(j-1)\left(p_{n}+q_{n}\right)+1}^{(j-1)\left(p_{n}+q_{n}\right)+p_{n}} X_{i}\right\|_{n \rightarrow \infty} 0 .
$$

Therefore, it is sufficient to prove the convergence in distribution to a Gaussian law of the second sum, which is easier than with the first one. Thus, Doukhan and Wintenberger [12] prove a $(2+\delta)$-order moment inequality which entails condition $A\left(k_{n}\right) \rightarrow 0$ and $T\left(k_{n}\right) \underset{k \rightarrow \infty}{\longrightarrow} 0$, and they obtain:

Theorem 2. Let $\left(X_{i}\right)_{i \in \mathbb{N}}$ be a sequence of stationary zero mean $(2+\delta)$-order random variables $($ with $\delta>0)$. Assume that $\left(X_{i}\right)_{i \in \mathbb{N}}$ is a $\lambda$ - (or $\theta$-) weakly dependent time series satisfying $\lambda_{r}=O\left(r^{-c}\right)$ (or $\left.\theta_{r}=O\left(r^{-c}\right)\right)$ when $r \rightarrow \infty$, with $c>4+2 / \delta$, then it exists $0<\sigma^{2}<\infty$ such that $\frac{1}{\sqrt{n}} \sum_{i=1}^{n} X_{i} \underset{k \rightarrow \infty}{\stackrel{\mathcal{D}}{\longrightarrow}} \mathcal{N}\left(0, \sigma^{2}\right)$.

Note that in Doukhan and Wintenberger [12] the Donsker principle is also proved.

\subsection{Subsampling}

Assume that $\left(X_{i}\right)_{i \in \mathbb{Z}}$ is a zero mean $(2+\delta)$-order stationary sequence for some $\delta>0$, with $\Sigma=\operatorname{Cov}\left(X_{0}\right)$. Then, for a sequence $\left(m_{n}\right)_{n \in \mathbb{N}}$ such that $m_{n} \underset{n \rightarrow \infty}{\longrightarrow} \infty$ and $k_{n}=\left[n / m_{n}\right] \underset{n \rightarrow \infty}{\longrightarrow} \infty$. We consider a subsample $\left(X_{m_{n}}, \ldots, X_{k_{n} m_{n}}\right)$ of $\left(X_{1}, \ldots, X_{n}\right)$, and the sample,

$$
\left(Y_{1, n}, \ldots, Y_{k_{n}, n}\right) \text { with } Y_{i, n}=\frac{1}{\sqrt{k_{n}}} X_{i m_{n}} \text { for } 1 \leq i \leq k_{n}
$$

Depending on the weak dependence property of $\left(X_{i}\right)_{i \in \mathbb{Z}}$, we can obtain the Lindeberg Theorem for

$$
S_{k_{n}, n}=\sum_{i=1}^{k_{n}} Y_{i, n}
$$


Proposition 4.1. Assume that $\left(X_{i}\right)_{i \in \mathbb{Z}}$ is a zero mean $(2+\delta)$-order stationary sequence for some $\delta>0$, with $\Sigma=\operatorname{Cov}\left(X_{0}\right)$. Then, for a sequence $\left(m_{n}\right)_{n \in \mathbb{N}}$ such that $m_{n} \underset{n \rightarrow \infty}{\longrightarrow} \infty$ and $k_{n}=\left[n / m_{n}\right] \underset{n \rightarrow \infty}{\longrightarrow} \infty$,

$$
S_{k_{n}, n}=\frac{1}{\sqrt{k_{n}}} \sum_{i=1}^{k_{n}} X_{i m_{n}} \underset{n \rightarrow \infty}{\stackrel{\mathcal{D}}{\longrightarrow}} \mathcal{N}_{d}(0, \Sigma),
$$

if one of the following assumptions also holds,

- $\left(X_{i}\right)_{i \in \mathbb{Z}}$ is a $\theta$-weakly dependent sequence and $\theta_{m_{n}} \sqrt{k_{n}} \underset{n \rightarrow \infty}{\longrightarrow} 0$.

- $\left(X_{i}\right)_{i \in \mathbb{Z}}$ is a $\lambda$-weakly dependent sequence and $\lambda_{m_{n}} k_{n}^{\frac{3}{2}} \underset{n \rightarrow \infty}{\longrightarrow} 0$.

This proposition allows to pass from a situation where the CLT is not satisfied to a situation where it is satisfied by using a subsample with the correct asymptotic step of sampling. For instance, if $X$ is a zero mean stationary long range dependent $(2+\delta$ )-order process (with $\delta>0)$ and such that a/ $X$ is a Gaussian process such that $\mathbb{E}\left(X_{0} X_{n}\right)=O\left(n^{2 H-2}\right)$ with $1 / 2<H<1$ when $n \rightarrow \infty$ or b/ $X$ a linear process. Then $X$ is a $\lambda$-weakly dependent process with $\lambda_{r}=O\left(r^{2 H-2}\right)$ and it is well known (see for instance Taqqu [20]) that $X$ does not satisfy a usual central limit theorem. As a consequence, with a subsampling step $m_{n}$ such that $o\left(m_{n}\right)=n^{3 /(4 H-1)}$, the subsampled time series $\left(X_{j m_{n}}\right)$ satisfies a usual CLT with a convergence rate $o\left(n^{(1-H) /(4 H-1)}\right)$.

Two objections can be raised to this method: first, only a part of the sample is used. The second and main objection is that the choice of the convergence rate of the subsampling implies the knowledge of the convergence rate of $\left(\lambda_{r}\right)$ or $\left(\theta_{r}\right)$. However, an estimation of this last rate (for instance in the case of long-range dependence) could provide an estimation of a fitted step of subsampling, or in the case of an exponential rate of convergence of $\left(\lambda_{r}\right)$ or $\left(\theta_{r}\right)$, all subsampling step $m_{n}=O\left(n^{a}\right)$ with $0<a<1$ could be used: it is the case for instance for $\operatorname{GARCH}(p, q)$ processes.

\subsection{Kernel density estimation}

Let $\left(X_{i}\right)_{i \in \mathbb{N}}$ be a sequence of stationary zero mean r.v. (with real values) such that $X_{0}$ has a marginal density $f_{X}$ with respect to Lebesgue measure. Let $K: \mathbb{R} \rightarrow \mathbb{R}$ be a kernel function satisfying,

Assumption $\mathbf{K}: K: \mathbb{R} \rightarrow \mathbb{R}$ be a bounded and Lipschitz function with $\int_{-\infty}^{\infty} K(t) \mathrm{d} t=1$.

Now, define $\left(h_{n}\right)_{n \in \mathbb{N}}$ a sequence such that $h_{n} \underset{n \rightarrow \infty}{\longrightarrow} 0$ and consider the usual kernel density estimation,

$$
\widehat{f}_{X}^{(n)}(x)=\frac{1}{n} \sum_{i=1}^{n} \frac{1}{h_{n}} K\left(\frac{x-X_{i}}{h_{n}}\right) \quad \text { for } x \in \mathbb{R}
$$

Proposition 4.2. Let $\left(X_{i}\right)_{i \in \mathbb{Z}}$ be a stationary zero mean weakly dependent time series (with real values) such that $X_{0}$ has a marginal density $f_{X}$ with respect to Lebesgue measure. Assume that $\left\|f_{X}\right\|_{\infty}<\infty$ and $\max _{i \neq j}\left\|f_{i, j}\right\|_{\infty}<\infty$, where $f_{i, j}$ denotes the joint marginal density of $\left(X_{i}, X_{j}\right)$. Then,

$$
\sqrt{n h_{n}}\left(\widehat{f}_{X}^{(n)}(x)-\mathbb{E} \widehat{f}_{X}^{(n)}(x)\right) \underset{n \rightarrow \infty}{\stackrel{\mathcal{D}}{\longrightarrow}} \mathcal{N}\left(0, f_{X}(x) \int_{\mathbb{R}} K^{2}(t) \mathrm{d} t\right),
$$

if $h_{n} \underset{n \rightarrow \infty}{\longrightarrow} 0, n h_{n} \underset{n \rightarrow \infty}{\longrightarrow} \infty$ and,

- $h_{n}=o\left(n^{-2 /\left(\lambda^{\prime}-4\right)} \wedge n^{-5 /\left(2 \lambda^{\prime}-5\right)}\right)$ when $\left(X_{i}\right)_{i \in \mathbb{Z}}$ is a $\lambda$-weakly dependent process with $\lambda_{r}=O\left(r^{-\lambda}\right)$ and $\lambda^{\prime}>\lambda>5$, or

- when $\left(X_{i}\right)_{i \in \mathbb{Z}}$ is a $\theta$-weakly dependent process with $\theta_{r}=O\left(r^{-\theta}\right)$ and $\theta>3$. 
Now, following the regularity of the function $f_{X}, \mathbb{E} \widehat{f}_{X}^{(n)}(x)$ is a more or less good approximation of $f_{X}(x)$. Hence, here there are two different cases of the approximation of the bias,

Corrolary 4.1. Assume that for some $p \in \mathbb{N}^{*}$, the function $f_{X}$ is a $\mathcal{C}^{p}(\mathbb{R})$ function, with bounded derivatives. Then, under the conditions of Proposition 4.2, with $K$ a kernel such that $\int_{\mathbb{R}} K(t) t^{q} \mathrm{~d} t=0$ for $q=\{1, \ldots, p-1\}$ and $\int_{\mathbb{R}} K(t) t^{p} \mathrm{~d} t \neq 0$, if $h_{n}=C \cdot n^{-1 /(2 p+1)}$ (with $C>0$ ) and, following the different frames of weak dependence, $\lambda>5 p+5$ or $\theta>3$,

$$
\sqrt{n h_{n}}\left(\widehat{f}_{X}^{(n)}(x)-f_{X}(x)\right) \underset{n \rightarrow \infty}{\stackrel{\mathcal{D}}{\longrightarrow}} \mathcal{N}\left(f_{X}^{(p)}(x) \frac{1}{p !} \int_{\mathbb{R}} t^{p} K(t) \mathrm{d} t, f_{X}(x) \int_{\mathbb{R}} K^{2}(t) \mathrm{d} t\right) .
$$

Corrolary 4.2. Assume that the regularity of the function $f_{X}$ is $\rho>0$ with $\rho \notin \mathbb{N}^{*}$ (in the sense that $f$ has a $[\rho]$ th order bounded derivative which is Hölder continuous with exponent $\rho-[\rho])$. Then, under the same conditions than Proposition 4.2, with $K$ a kernel such that $\int_{\mathbb{R}} K(t) t^{q} d t=0$ for $q=\{1, \ldots,[\rho]-1\}$ and $\int_{\mathbb{R}} K(t) t^{[\rho]} d t \neq 0$, if $h_{n}=o\left(n^{-1 /(2[\rho]+1)}\right)$ and, following the different frames of weak dependence, $\lambda>5[\rho]+5$ or $\theta>3$,

$$
\sqrt{n h_{n}}\left(\widehat{f}_{X}^{(n)}(x)-f_{X}(x)\right) \underset{n \rightarrow \infty}{\stackrel{\mathcal{D}}{\longrightarrow}} \mathcal{N}\left(0, f_{X}(x) \int_{\mathbb{R}} K^{2}(t) \mathrm{d} t\right) .
$$

\subsection{Subsampled kernel density estimation}

Now, imagine that the process $\left(X_{k}\right)_{k \in \mathbb{Z}}$ is a weakly dependent zero mean stationary process such that the conditions $\lambda>5$ or $\theta>3$ of Proposition 4.2 are not satisfied. As a consequence, the kernel density estimator is not proved to satisfy a central limit theorem. Subsampling can provide a way for obtaining a CLT (and then, confidence intervals or goodness-of-fit tests). Indeed, like it was also considered before, a subsampled time series with an asymptotic rate is "less" dependent than the original time series. Indeed, consider a sequence $\left(m_{n}\right)_{n \in \mathbb{N}}$ such that

$$
m_{n} \underset{n \rightarrow \infty}{\longrightarrow} \infty \text { and } k_{n}=\left[n / m_{n}\right] \underset{n \rightarrow \infty}{\longrightarrow} \infty
$$

and the subsample $\left(X_{m_{n}}, \ldots, X_{k_{n} m_{n}}\right)$ of $\left(X_{1}, \ldots, X_{n}\right)$. For $\left(h_{n}\right)_{n \in \mathbb{N}}$ a sequence such that $h_{n} \underset{n \rightarrow \infty}{\longrightarrow} 0$, define the subsampled kernel density estimator of $f_{X}$ as:

$$
\widehat{f}_{X}^{\left(n, m_{n}\right)}(x)=\frac{1}{k_{n}} \sum_{i=1}^{k_{n}} \frac{1}{h_{n}} K\left(\frac{x-X_{i m_{n}}}{h_{n}}\right) \quad \text { for } x \in \mathbb{R} .
$$

Proposition 4.3. Under the same assumptions than Proposition 4.2, except that $0 \leq \lambda \leq 6$ or $\theta \leq 3$, the following CLT yields from the subsample $\left(X_{m_{n}}, \ldots, X_{k_{n} m_{n}}\right)$,

$$
\sqrt{k_{n} h_{n}}\left(\widehat{f}_{X}^{\left(n, m_{n}\right)}(x)-\mathbb{E} \widehat{f}_{X}^{\left(n, m_{n}\right)}(x)\right) \underset{n \rightarrow \infty}{\stackrel{\mathcal{D}}{\longrightarrow}} \mathcal{N}\left(0, f_{X}(x) \int_{\mathbb{R}} K^{2}(t) \mathrm{d} t\right)
$$

for sequences $\left(h_{n}\right)$ and $\left(m_{n}\right)$ such that $h_{n}=n^{-h}$ and $m_{n}=n^{m}$, where the points $(m, h)$ are in the "white zonas" (included in square $\left.(0,1)^{2}\right)$ of Figures 1 and 2 (see below). Moreover, the "optimal" convergence rate is obtained for,

- $\sqrt{h_{n} k_{n}}=n^{\frac{\lambda}{5+2(\lambda \vee 1)}-\varepsilon}$ for all $\varepsilon>0$ small enough (and $h_{n}=n^{-\varepsilon}$ and $m_{n}=n^{\frac{5}{5+2(\lambda \vee 1)}+\varepsilon}$ ) when $\left(X_{i}\right)_{i \in \mathbb{Z}}$ is a $\lambda$-weakly dependent process;

- $\sqrt{h_{n} k_{n}}=n^{\frac{1}{2}(\theta \wedge 1)-\varepsilon}$ for all $\varepsilon>0$ small enough (and $h_{n}=n^{-\varepsilon}$ and $m_{n}=n^{((1-\theta) \vee 0)+\varepsilon}$ ) when $\left(X_{i}\right)_{i \in \mathbb{Z}}$ is a $\theta$-weakly dependent process.

From this result, we now answer to the following problem. Assume that $X$ is a $\theta$ - (or $\lambda$-) weakly dependent process and that the regularity $\rho$ (in the sense of the previous section) of the density function $f_{X}$ and the 

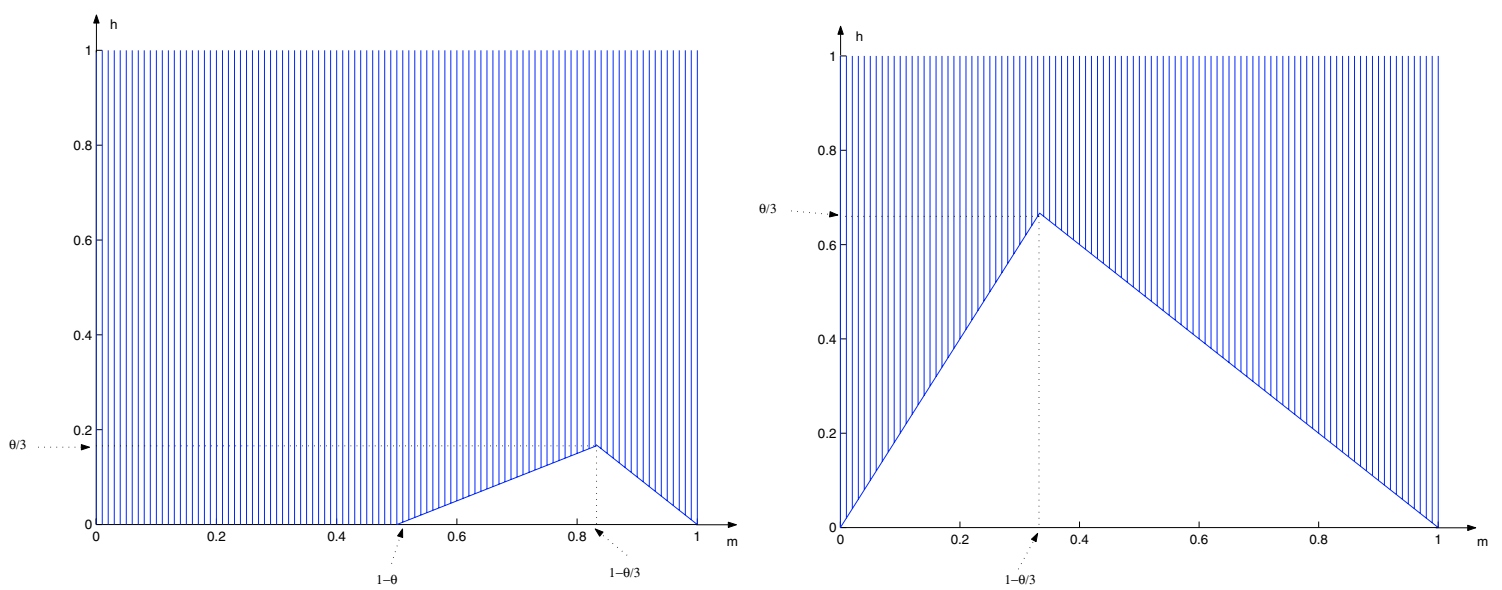

Figure 1. Conditions (the "white" zona) satisfied by parameters $m$ and $h$ for obtaining the CLT in the $\theta$-weak dependence frame, when $0<\theta \leq 1$ (left) and $1<\theta \leq 3$ (right).
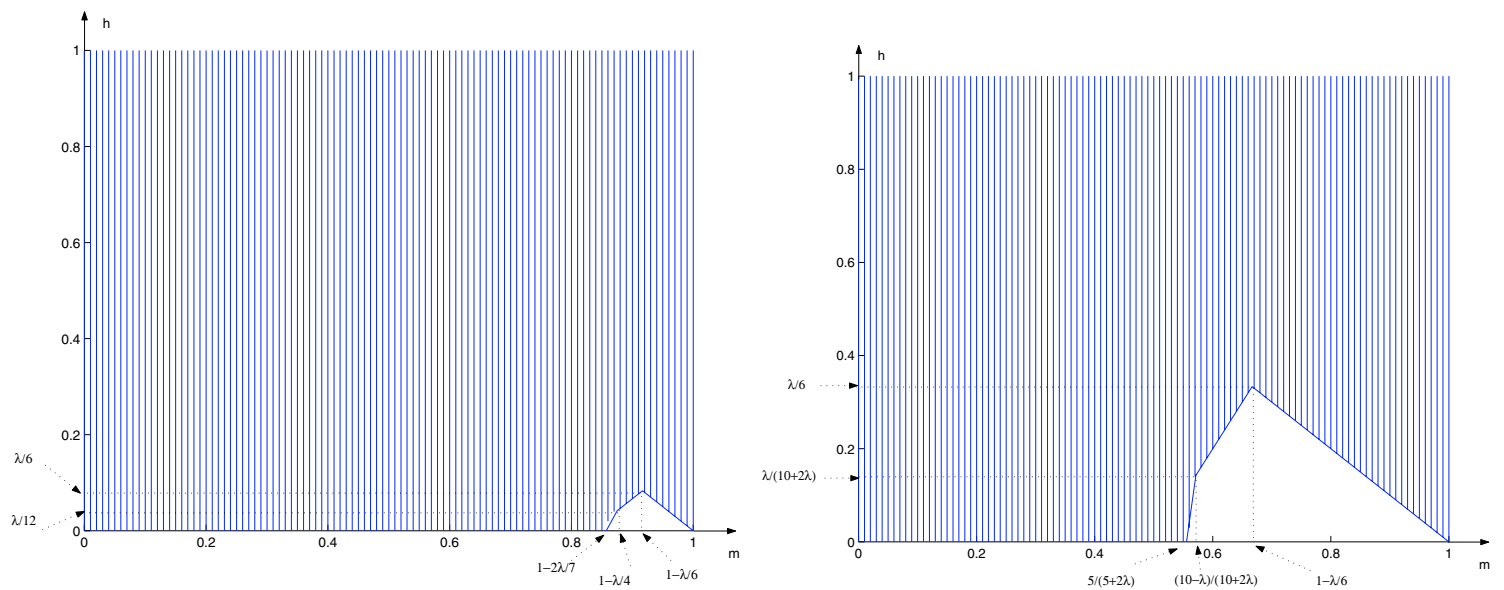

Figure 2. Conditions (the "white" zona) satisfied by parameters $m$ and $h$ for obtaining the CLT in the $\lambda$-weak dependence frame, when $0<\lambda \leq 1$ (left) and $1<\lambda \leq 6$ (right).

sequence decay rates $\theta$ and $\lambda$ of the sequences $\left(\theta_{r}\right)_{r}$ or $\left(\lambda_{r}\right)_{r}$ are known. In the previous section, we have established a CLT for the density kernel estimator when $\theta$ (or $\lambda$ ) is larger than an affine function of $\rho$. However, if this inequality is not satisfied, it is possible to find a subsampling step such that a CLT for the subsampled kernel density estimator can be proved, Proposition 4.2. We have also,

Corrolary 4.3. Under the assumptions of consequence 4.1, but if $0<\lambda \leq 5 p+5$ (or $0<\theta \leq p+3)$,

$$
\sqrt{k_{n} h_{n}}\left(\widehat{f}_{X}^{\left(n, m_{n}\right)}(x)-f_{X}(x)\right) \underset{n \rightarrow \infty}{\stackrel{\mathcal{D}}{\longrightarrow}} \mathcal{N}\left(f_{X}^{(p)}(x) \frac{1}{p !} \int_{\mathbb{R}} t^{p} K(t) \mathrm{d} t, f_{X}(x) \int_{\mathbb{R}} K^{2}(t) \mathrm{d} t\right)
$$

with the following optimal conditions,

- for $\lambda$-weakly dependent process, with $\lambda_{r}=O\left(r^{-\lambda}\right)$ and $0<\lambda \leq 5 p+5$, for $h_{n}=n^{-\frac{\lambda}{5+p(5+2(\lambda \vee 1))}}$ and $m_{n}=n^{1-\frac{\lambda(2 p+1)}{5+p(5+2(\lambda \vee 1))}}$. Then, the convergence rate is $\sqrt{k_{n} h_{n}} \sim n^{\frac{p \lambda}{5+p(5+2(\lambda \vee 1))}}$; 
- for $\theta$-weakly dependent process, with $\theta_{r}=O\left(r^{-\theta}\right)$ and $0<\theta \leq p+3$, for $h_{n}=n^{-\frac{\theta}{3+2 p(\theta \vee 1)}}$ and $m_{n}=$ $n^{1-\frac{\theta(2 p+1)}{3+2 p(\theta \vee 1)}}$. Then, the convergence rate is $\sqrt{k_{n} h_{n}} \sim n^{\frac{p \theta}{3+2 p(\theta \vee 1)}}$.

Corrolary 4.4. Under the assumptions of Consequence 4.2 with $\rho \notin \mathbb{N}$, but if $0<\lambda \leq 5[\rho]+5$ (or $0<\theta \leq$ $[\rho]+3)$, then,

$$
\sqrt{k_{n} h_{n}}\left(\widehat{f}_{X}^{\left(n, m_{n}\right)}(x)-f_{X}(x)\right) \underset{n \rightarrow \infty}{\stackrel{\mathcal{D}}{\longrightarrow}} \mathcal{N}\left(0, f_{X}(x) \int_{\mathbb{R}} K^{2}(t) \mathrm{d} t\right),
$$

with the following conditions (for all $\varepsilon>0$ small enough),

- for $\lambda$-weakly dependent process, with $\lambda_{r}=O\left(r^{-\lambda}\right)$ and $0<\lambda \leq 5[\rho]+5$, for $h_{n}=n^{-\frac{\lambda}{5+[\rho](5+2(\lambda \vee 1))}}$ and $m_{n}=n^{1+2 \varepsilon-\frac{\lambda(2[\rho]+1)}{5+[\rho(5+2(\lambda \vee 1))}}$. Then, the convergence rate is $\sqrt{k_{n} h_{n}} \sim n^{\frac{\rho \lambda}{5+[\rho](5+2(\lambda \vee 1))}}{ }^{-\varepsilon}$;

- for $\theta$-weakly dependent process, with $\theta_{r}=O\left(r^{-\theta}\right)$ and $0<\theta \leq[\rho]+3$, for $h_{n}=n^{-\frac{\theta}{3+2[\rho](\theta \vee 1)}}$ and $m_{n}=n^{1+2 \varepsilon-\frac{\theta(2[\rho]+1)}{3+2[\rho](\theta \vee 1)}}$. Then, the convergence rate is $\sqrt{k_{n} h_{n}} \sim n^{\frac{[\rho \rho] \theta}{3+2[\rho](\theta \vee 1)}-\varepsilon}$.

Hence, for all regularity parameter $\rho>0$, even if $\lambda$ or $\theta$ are very small numbers (for instance when $X$ is a long range dependent process), the subsampled kernel density estimator satisfies a CLT for a fitted choice of $h_{n}$ and $m_{n}$. Moreover, for $\theta$-weakly dependent time series with $\theta>0$, when $\rho \rightarrow \infty$, the convergence rate of this theorem is $n^{1 / 2-\varepsilon}$, with $\varepsilon>0$.

\section{PROOFS}

Proof of Lemma 1. For $k \in \mathbb{N}^{*}$, we first notice and prove that,

$$
\begin{aligned}
\Delta_{k} & \leq \Delta_{k, 1}+\cdots+\Delta_{k, k} \\
\text { with } \Delta_{k, i} & =\left|\mathbb{E}\left(f_{i}\left(W_{i}+X_{i}\right)-f_{i}\left(W_{i}+Y_{i}\right)\right)\right|, \quad \text { for } i \in\{1, \ldots, k\} \\
W_{i} & =X_{1}+\cdots+X_{i-1} \quad \text { and } W_{1}=0, \text { for } i \in\{2, \ldots, k\}, \\
f_{i}(t) & =\mathbb{E}\left(f\left(t+Y_{i+1}+\cdots+Y_{k}\right)\right) \text { and } f_{k}(t)=f(t), \text { for } t \in \mathbb{R}^{d} \text { and } i \in\{1, \ldots, k-1\} .
\end{aligned}
$$

Let $x, w \in \mathbb{R}^{d}$. Taylor formula writes in two following ways (for suitable vectors $w_{1, x}, w_{2, x} \in \mathbb{R}^{d}$ ),

$$
\begin{aligned}
f(w+x) & =f(w)+f^{(1)}(w)(x)+\frac{1}{2} f^{(2)}\left(w_{1, x}\right)(x, x) \\
& =f(w)+f^{(1)}(w)(x)+\frac{1}{2} f^{(2)}(w)(x, x)+\frac{1}{6} f^{(3)}\left(w_{2, x}\right)(x, x, x),
\end{aligned}
$$

where, for $j=1,2$ and $3, f^{(j)}(w)\left(y_{1}, \ldots, y_{j}\right)$ stands for the value of the symmetric $j$-linear form $f^{(j)}$ of $\left(y_{1}, \ldots, y_{j}\right)$ at $w$. Moreover, denote,

$$
\left\|f^{(j)}(w)\right\|_{1}=\sup _{\left\|y_{1}\right\|, \ldots,\left\|y_{j}\right\| \leq 1}\left|f^{(j)}(w)\left(y_{1}, \ldots, y_{j}\right)\right|, \quad\left\|f^{(j)}\right\|_{\infty}=\sup _{w \in \mathbb{R}^{d}}\left\|f^{(j)}(w)\right\|_{1} .
$$

Thus for $w, x, y \in \mathbb{R}^{d}$, there exists some suitable vectors $w_{1, x}, w_{2, x}, w_{1, y}, w_{2, y} \in \mathbb{R}^{d}$ such that:

$$
\begin{aligned}
f(w+x)-f(w+y)= & f^{(1)}(w)(x-y)+\frac{1}{2}\left(f^{(2)}(w)(x, x)-f^{(2)}(w)(y, y)\right) \\
& +\frac{1}{2}\left(\left(f^{(2)}\left(w_{1, x}\right)-f^{(2)}(w)\right)(x, x)-\left(f^{(2)}\left(w_{1, y}\right)-f^{(2)}(w)\right)(y, y)\right) \\
= & f^{(1)}(w)(x-y)+\frac{1}{2}\left(f^{(2)}(w)(x, x)-f^{(2)}(w)(y, y)\right) \\
& +\frac{1}{6}\left(f^{(3)}\left(w_{2, x}\right)(x, x, x)-f^{(3)}\left(w_{2, y}\right)(y, y, y)\right) .
\end{aligned}
$$


Thus, $\gamma=f(w+x)-f(w+y)-f^{(1)}(w)(x-y)-\frac{1}{2}\left(f^{(2)}(w)(x, x)-f^{(2)}(w)(y, y)\right) \quad$ satisfies

$$
\begin{aligned}
|\gamma| & \leq\left(\left(\|x\|^{2}+\|y\|^{2}\right)\left\|f^{(2)}\right\|_{\infty}\right) \wedge\left(\frac{1}{6}\left(\|x\|^{3}+\|y\|^{3}\right)\left\|f^{(3)}\right\|_{\infty}\right) \\
& \leq\left(\|x\|^{2}\left\|f^{(2)}\right\|_{\infty}\right) \wedge\left(\frac{1}{6}\|x\|^{3}\left\|f^{(3)}\right\|_{\infty}\right)+\left(\|x\|^{2}\left\|f^{(2)}\right\|_{\infty}\right) \wedge\left(\frac{1}{6}\|y\|^{3}\left\|f^{(3)}\right\|_{\infty}\right) \\
& +\left(\|y\|^{2}\left\|f^{(2)}\right\|_{\infty}\right) \wedge\left(\frac{1}{6}\|x\|^{3}\left\|f^{(3)}\right\|_{\infty}\right)+\left(\|y\|^{2}\left\|f^{(2)}\right\|_{\infty}\right) \wedge\left(\frac{1}{6}\|y\|^{3}\left\|f^{(3)}\right\|_{\infty}\right) \\
& \leq \frac{1}{6^{\delta}}\left\|f^{(2)}\right\|_{\infty}^{1-\delta}\left\|f^{(3)}\right\|_{\infty}^{\delta}\left\{\|x\|^{2+\delta}+\|x\|^{2(1-\delta)}\|y\|^{3 \delta}+\|y\|^{2(1-\delta)}\|x\|^{3 \delta}+\|y\|^{2+\delta}\right\}
\end{aligned}
$$

using the inequality $1 \wedge c \leq c^{\delta}$, that is valid for all $c \geq 0$ when $\delta \in[0,1]$.

Substituting $f_{i}, W_{i}, X_{i}$ and $Y_{i}$ to $f, w, y$ and $x$ in the preceding inequality and taking expectation gives a bound to $\Delta_{k, i}$. Indeed,

$$
\begin{aligned}
\mathbb{E} f_{i}\left(W_{i}+\right. & \left.X_{i}\right)-\mathbb{E} f_{i}\left(W_{i}+Y_{i}\right) \\
& =\mathbb{E} f_{i}\left(W_{i}+X_{i}\right)-\mathbb{E} f_{i}\left(W_{i}+Y_{i}\right)-\mathbb{E}\left(f_{i}^{(1)}\left(W_{i}\right)\left(X_{i}-Y_{i}\right)-\frac{1}{2}\left(f_{i}^{(2)}\left(W_{i}\right)\left(X_{i}, X_{i}\right)-f_{i}^{(2)}\left(W_{i}\right)\left(Y_{i}, Y_{i}\right)\right)\right)
\end{aligned}
$$

because $W_{i}$ is independent from $X_{i}$ and $Y_{i}$ and because $\mathbb{E} X_{i}=\mathbb{E} Y_{i}=0$ and $\operatorname{Cov} X_{i}=\operatorname{Cov} Y_{i}$. Using Jensen's inequality and the independence of $X$ 's and $Y^{\prime}$ 's, we derive $\mathbb{E}\left(\left\|Y_{i}\right\|^{2+\delta}\right) \leq\left(\mathbb{E}\left(\left\|Y_{i}\right\|^{4}\right)\right)^{\frac{1}{2}+\frac{\delta}{4}}$ and $\mathbb{E}\left(\left\|Y_{i}\right\|^{4}\right) \leq$ $3 \cdot \mathbb{E}^{2}\left(\left\|X_{i}\right\|^{2}\right)$ because $Y_{i}$ is a Gaussian r.v. with the same covariance than $X_{i}$. Therefore

$$
\begin{array}{llll}
\mathbb{E}\left(\left\|Y_{i}\right\|^{2+\delta}\right) & \leq 3^{\frac{1}{2}+\frac{\delta}{4}}\left(\mathbb{E}\left(\left\|X_{i}\right\|^{2+\delta}\right)\right)^{4\left(\frac{1}{2}+\frac{\delta}{4}\right) /(2+\delta)} & \leq 3^{\frac{1}{2}+\frac{\delta}{4}} \mathbb{E}\left(\left\|X_{i}\right\|^{2+\delta}\right) & \\
\mathbb{E}\left\|X_{i}\right\|^{2(1-\delta)} \mathbb{E}\left\|Y_{i}\right\|^{3 \delta} & \leq \mathbb{E}\left\|X_{i}\right\|^{2(1-\delta)}\left(\mathbb{E}\left\|X_{i}\right\|^{2}\right)^{3 \delta / 2} & \leq \mathbb{E}\left\|X_{i}\right\|^{2+\delta} & \text { for } 3 \delta \leq 2 \\
& \leq 3^{3 \delta / 4} \mathbb{E}\left\|X_{i}\right\|^{2(1-\delta)}\left(\mathbb{E}\left\|X_{i}\right\|^{2}\right)^{3 \delta / 2} & \leq 3^{\frac{1}{2}+\frac{\delta}{4}} \mathbb{E}\left\|X_{i}\right\|^{2+\delta} & \text { else } \\
\mathbb{E}\left\|Y_{i}\right\|^{2(1-\delta)} \mathbb{E}\left\|X_{i}\right\|^{3 \delta} & \leq\left(\mathbb{E}\left\|Y_{i}\right\|^{2}\right)^{1-\delta} \mathbb{E}\left\|X_{i}\right\|^{3 \delta} & \leq\left(\mathbb{E}\left\|X_{i}\right\|^{2}\right)^{1-\delta} \mathbb{E}\left\|X_{i}\right\|^{3 \delta} \leq \mathbb{E}\left\|X_{i}\right\|^{2+\delta}
\end{array}
$$

in the case $3 \delta>2$ (we note that $3 \delta \leq 4$ ) the second inequality relies on the relations $\left\|Y_{i}\right\|_{3 \delta} \leq\left\|Y_{i}\right\|_{4} \leq 3^{1 / 4}\left\|X_{i}\right\|_{2}$. Those inequalities allow to consider the terms between braces in (9). Recall that $\left\|f_{i}^{(j)}\right\|_{\infty} \leq\left\|f^{(j)}\right\|_{\infty}$ ( for $1 \leq i \leq k$ and $0 \leq j \leq 3)$. We obtain

$$
\Delta_{k, i} \leq \frac{2\left(1+3^{\frac{1}{2}+\frac{\delta}{4}}\right)}{6^{\delta}}\left\|f^{(2)}\right\|_{\infty}^{1-\delta}\left\|f^{(3)}\right\|_{\infty}^{\delta} \mathbb{E}\left\|X_{i}\right\|^{2+\delta} \leq 6\left\|f^{(2)}\right\|_{\infty}^{1-\delta}\left\|f^{(3)}\right\|_{\infty}^{\delta} \mathbb{E}\left\|X_{i}\right\|^{2+\delta}
$$

As a consequence, from assumption (2),

$$
\Delta_{k} \leq 6 \cdot A_{k} \cdot\left\|f^{(2)}\right\|_{\infty}^{1-\delta}\left\|f^{(3)}\right\|_{\infty}^{\delta}
$$

Proof of Remark 1. Set $b_{k}^{2}=\max _{1 \leq i \leq k} \mathbb{E}\left(\left\|X_{i}\right\|^{2}\right)$. Now, for $\varepsilon<6\left\|f^{(2)}\right\|_{\infty} \cdot\left(\left\|f^{(3)}\right\|_{\infty}\right)^{-1}$, and using inequality (9), one obtains

$$
\begin{aligned}
\Delta_{k, i} & \leq\left\|f^{(2)}\right\|_{\infty} \mathbb{E}\left(\left\|X_{i}\right\|^{2} \wedge \frac{1}{6}\left(\left\|f^{(2)}\right\|_{\infty}\right)^{-1}\left\|f^{(3)}\right\|_{\infty} \cdot\left\|X_{i}\right\|^{3}\right)+\frac{1}{3}\left\|f^{(3)}\right\|_{\infty} \mathbb{E}\left(\left\|Y_{i}\right\|^{3}\right) \\
& \left.+\left\|f^{(2)}\right\|_{\infty} \mathbb{E}\left(\left\|Y_{i}\right\|^{2} \wedge \frac{1}{6}\left(\left\|f^{(2)}\right\|_{\infty}\right)^{-1}\left\|f^{(3)}\right\|_{\infty} \cdot\left\|X_{i}\right\|^{3}\right)\right) \\
& \leq\left\|f^{(2)}\right\|_{\infty} \mathbb{E}\left(\left\|X_{i}\right\|^{2} \mathbb{1}_{\left\{\left\|X_{i}\right\|>\varepsilon\right\}}\right)+\frac{1}{3}\left\|f^{(3)}\right\|_{\infty} \cdot\left(\mathbb{E}\left(\left\|X_{i}\right\|^{3} \mathbb{1}_{\left\{\left\|X_{i}\right\| \leq \varepsilon\right\}}\right)+\mathbb{E}\left(\left\|Y_{i}\right\|^{3}\right)\right) \\
& +\left\|f^{(2)}\right\|_{\infty} \mathbb{E}\left(\left\|Y_{i}\right\|^{2} \mathbb{1}_{\left\{\left\|X_{i}\right\|>\varepsilon\right\}}\right) .
\end{aligned}
$$


Now as any nonnegative square integrable real valued r.v. $Z$ is associated, we get

$$
\left.\operatorname{Cov}\left(Z^{2}, \mathbb{1}_{\{Z>\varepsilon\}}\right)=\mathbb{E} Z^{2} \mathbb{1}_{\{Z>\varepsilon\}}\right)-\mathbb{E} Z^{2} \mathbb{P}(Z>\varepsilon) \geq 0
$$

thus

$$
\mathbb{E}\left(\left\|Y_{i}\right\|^{2} \mathbb{1}_{\left\{\left\|X_{i}\right\|>\varepsilon\right\}}\right)=\mathbb{E}\left\|Y_{i}\right\|^{2} \cdot \mathbb{P}\left(\left\|X_{i}\right\|>\varepsilon\right)=\mathbb{E}\left\|X_{i}\right\|^{2} \cdot \mathbb{P}\left(\left\|X_{i}\right\|>\varepsilon\right) \leq \mathbb{E}\left(\left\|X_{i}\right\|^{2} \mathbb{1}_{\left\{\left\|X_{i}\right\|>\varepsilon\right\}}\right)
$$

so that

$$
\Delta_{k, i} \leq 2\left\|f^{(2)}\right\|_{\infty} \mathbb{E}\left(\left\|X_{i}\right\|^{2} \mathbb{1}_{\left\{\left\|X_{i}\right\|>\varepsilon\right\}}\right)+\frac{1}{3}\left\|f^{(3)}\right\|_{\infty} \cdot\left(\varepsilon \cdot \mathbb{E}\left(\left\|X_{i}\right\|^{2}\right)+3^{3 / 4}\left(\mathbb{E}\left(\left(\left\|X_{i}\right\|^{2}\right)^{3 / 2}\right)\right),\right.
$$

from the Hölder Inequality. It implies that

$$
\begin{aligned}
\Delta_{k} & \leq 2\left\|f^{(2)}\right\|_{\infty} B_{k}(\varepsilon)+\frac{1}{3}\left\|f^{(3)}\right\|_{\infty}\left(\varepsilon \cdot a_{k}+3^{3 / 4} \sum_{i=1}^{k} b_{k} \cdot \mathbb{E}\left(\left\|X_{i}\right\|^{2}\right)\right) \\
& \leq 2\left\|f^{(2)}\right\|_{\infty} B_{k}(\varepsilon)+\frac{1}{3}\left\|f^{(3)}\right\|_{\infty} \cdot a_{k}\left(\varepsilon+3^{3 / 4} \cdot b_{k}\right) .
\end{aligned}
$$

Moreover, $b_{k}^{2} \leq \varepsilon^{2}+\max _{1 \leq i \leq k} \mathbb{E}\left(\left\|X_{i}\right\|^{2} \mathbb{1}_{\left\{\left\|X_{i}\right\|>\varepsilon\right\}}\right)$, therefore $b_{k}^{2} \leq \varepsilon^{2}+B_{k}(\varepsilon)$ and thus $b_{k} \leq \varepsilon+\sqrt{B_{k}(\varepsilon)}$. As a consequence,

$$
\Delta_{k} \leq 2\left\|f^{(2)}\right\|_{\infty} B_{k}(\varepsilon)+\left\|f^{(3)}\right\|_{\infty} \cdot a_{k}\left(\frac{4}{3} \varepsilon+\sqrt{B_{k}(\varepsilon)}\right) .
$$

Proof of Lemma 2. Consider $\left(X_{i}^{*}\right)_{i \in \mathbb{N}}$ a sequence of r.v. satisfying Assumption $H_{\delta}$ and such that $\left(X_{i}^{*}\right)_{i \in \mathbb{N}}$ is independent of $\left(X_{i}\right)_{i \in \mathbb{N}}$ and $\left(Y_{i}\right)_{i \in \mathbb{N}}$. Moreover, assume that $X_{i}^{*}$ has the same distribution as $X_{i}$ for $i \in \mathbb{N}$. Then, using the same decomposition as in the proof of Lemma 1 , one can also write,

$$
\left.\Delta_{k, i} \leq\left|\mathbb{E}\left(f_{i}\left(W_{i}+X_{i}\right)-f_{i}\left(W_{i}+X_{i}^{*}\right)\right)\right|+\mid \mathbb{E}\left(f_{i}\left(W_{i}+X_{i}^{*}\right)-f_{i}\left(W_{i}+Y_{i}\right)\right)\right) \mid .
$$

From the previous Lemma,

$$
\sum_{i=1}^{k}\left|\mathbb{E}\left(f_{i}\left(W_{i}+X_{i}^{*}\right)-f_{i}\left(W_{i}+Y_{i}\right)\right)\right| \leq \frac{2\left(1+3^{\frac{1}{2}+\frac{\delta}{4}}\right)}{6^{\delta}} \cdot\left\|f^{(2)}\right\|_{\infty}^{1-\delta} \cdot\left\|f^{(3)}\right\|_{\infty}^{\delta} \cdot A_{k} .
$$

Moreover, the bound for $\gamma$ of the proof of Lemma 1 entails,

$$
\begin{aligned}
& \left|f_{i}\left(W_{i}+X_{i}\right)-f_{i}\left(W_{i}+X_{i}^{*}\right)\right| \leq\left|f_{i}^{(1)}\left(W_{i}\right)\left(X_{i}-X_{i}^{*}\right)\right| \\
& \quad+\frac{1}{2}\left|f_{i}^{(2)}\left(W_{i}\right)\left(X_{i}, X_{i}\right)-f_{i}^{(2)}\left(W_{i}\right)\left(X_{i}^{*}, X_{i}^{*}\right)\right|+\frac{2}{6^{\delta}}\left\|f^{(2)}\right\|_{\infty}^{1-\delta}\left\|f^{(3)}\right\|_{\infty}^{\delta}\left\{\left\|X_{i}\right\|^{2+\delta}+\left\|X_{i}^{*}\right\|^{2+\delta}\right\},
\end{aligned}
$$

because $\left(X_{i}\right)$ is now supposed to be a dependent sequence of random variables and is no more independent from $\left(W_{i}\right)$. But with the notation before Lemma 2, we may write

$$
\begin{aligned}
& \sum_{i=1}^{k}\left|\mathbb{E} f_{i}^{(1)}\left(W_{i}\right)\left(X_{i}-X_{i}^{*}\right)\right|=\sum_{i=1}^{k}\left|\mathbb{E} f_{i}^{(1)}\left(W_{i}\right)\left(X_{i}\right)\right|=T_{1}, \quad \text { and } \\
& \sum_{i=1}^{k}\left|\mathbb{E}\left(f_{i}^{(2)}\left(W_{i}\right)\left(X_{i}, X_{i}\right)-f_{i}^{(2)}\left(W_{i}\right)\left(X_{i}^{*}, X_{i}^{*}\right)\right)\right|=T_{2} .
\end{aligned}
$$


It implies that,

$$
\Delta_{k} \leq T_{1}+\frac{1}{2} T_{2}+10 \cdot\left\|f^{(2)}\right\|_{\infty}^{1-\delta} \cdot\left\|f^{(3)}\right\|_{\infty}^{\delta} \cdot A_{k}
$$

Proof of Lemma 3. Here, for $t \in \mathbb{R}^{d}, f(x)=\mathrm{e}^{i\langle t, x\rangle}$. Denote $V_{i}=\operatorname{Var} X_{i}$, the covariance matrix of the vector $X_{i}$. Then, for a r.v. $Z$ independent from $\left(Y_{i}\right)_{i \in \mathbb{N}}$,

$$
\mathbb{E} f_{j}(Z)=\mathbb{E}\left(f\left(Z+Y_{j+1}+\cdots+Y_{k}\right)\right)=\mathrm{e}^{-\frac{1}{2} t^{\prime} \cdot\left(V_{j+1}+\cdots+V_{k}\right) \cdot t} \cdot \mathbb{E}\left(\mathrm{e}^{i\langle t, Z\rangle}\right) .
$$

Then, again

$$
\Delta_{k, j} \leq\left|\mathbb{E}\left(f_{j}\left(W_{j}+X_{j}\right)-f_{j}\left(W_{j}+X_{j}^{*}\right)\right)\right|+\left|\mathbb{E}\left(f_{j}\left(W_{j}+X_{j}^{*}\right)-f_{j}\left(W_{j}+Y_{j}\right)\right)\right|
$$

with the second term bounded as in the proof of Lemma 2 with $\left\|f^{(2)}\right\|_{\infty}^{1-\delta} \cdot\left\|f^{(3)}\right\|_{\infty}^{\delta}=\|t\|^{2+\delta}$, and for the first term,

$$
\begin{aligned}
\mathbb{E}\left(f_{j}\left(W_{j}+X_{j}\right)-f_{j}\left(W_{j}+X_{j}^{*}\right)\right) & =\mathrm{e}^{-\frac{1}{2} t^{\prime} \cdot\left(V_{j+1}+\cdots+V_{k}\right) \cdot t} \cdot \mathbb{E}\left(\mathrm{e}^{i\left\langle t, W_{j}\right\rangle}\left(\mathrm{e}^{i\left\langle t, X_{j}\right\rangle}-\mathrm{e}^{i\left\langle t, X_{j}^{*}\right\rangle}\right)\right), \\
\left|\mathbb{E}\left(f_{j}\left(W_{j}+X_{j}\right)-f_{j}\left(W_{j}+X_{j}^{*}\right)\right)\right| & \leq\left|\mathrm{e}^{-\frac{1}{2} t^{\prime} \cdot\left(V_{j+1}+\cdots+V_{k}\right) \cdot t}\right|\left|\operatorname{Cov}\left(\mathrm{e}^{i\left\langle t, W_{j}\right\rangle}, \mathrm{e}^{i\left\langle t, X_{j}\right\rangle}\right)\right|, \\
& \leq\left|\operatorname{Cov}\left(\mathrm{e}^{i\left\langle t, W_{j}\right\rangle}, \mathrm{e}^{i\left\langle t, X_{j}\right\rangle}\right)\right| .
\end{aligned}
$$

Proof of Proposition 4.1. The proof of this proposition is a consequence of Theorem 1, using $T(n) \underset{n \rightarrow \infty}{\longrightarrow} 0$. In one hand, thanks to the stationarity of the sequence $\left(X_{i}\right)_{i \in \mathbb{Z}}$,

$$
A_{k_{n}}=\sum_{i=1}^{k_{n}} \mathbb{E}\left(\left\|Y_{i, n}\right\|^{2+\delta}\right)=k_{n}^{-\delta / 2} \mathbb{E}\left(\left\|X_{0}\right\|^{2+\delta}\right) \underset{n \rightarrow \infty}{\longrightarrow} 0 .
$$

In the other hand, a bound of $T\left(k_{n}\right)$ can be provided. Indeed, let $t \in \mathbb{R}^{d}$, and then,

$$
\begin{aligned}
T\left(k_{n}\right) & =\sum_{j=1}^{k_{n}}\left|\operatorname{Cov}\left(\mathrm{e}^{i\left\langle t, Y_{1, n}+\cdots+Y_{j-1, n}\right\rangle}, \mathrm{e}^{i\left\langle t, Y_{j, n}\right\rangle}\right)\right| \\
& =\sum_{j=1}^{k_{n}}\left|\operatorname{Cov}\left(F_{t, n}\left(X_{m_{n}}, \ldots, X_{(j-1) m_{n}}\right), G_{t, n}\left(X_{j m_{n}}\right)\right)\right|
\end{aligned}
$$

with $G_{t, n}(s)=\mathrm{e}^{\left(i\langle s, t\rangle / \sqrt{k_{n}}\right)}$ and $F_{t, n}\left(s_{1}, \ldots, s_{j-1}\right)=G_{t, n}\left(s_{1}\right) \times \cdots \times G_{t, n}\left(s_{j-1}\right)$ for $\left(s_{1}, \ldots, s_{j}\right) \in\left(\mathbb{R}^{d}\right)^{j}$. But,

$$
\begin{array}{lll}
\left\|G_{t, n}\right\|_{\infty} \leq 1 & \text { and } & \operatorname{Lip} G_{t, n} \leq\|t\| \cdot k_{n}^{-1 / 2} \\
\left\|F_{t, n}\right\|_{\infty} \leq 1 & \text { and } & \operatorname{Lip} F_{t, n} \leq\|t\| \cdot k_{n}^{-1 / 2},
\end{array}
$$

from inequality $\left|u_{1} \times \cdots \times u_{j-1}-v_{1} \times \cdots \times v_{j-1}\right| \leq\left|u_{1}-v_{1}\right|+\cdots+\left|u_{j-1}-v_{j-1}\right|$, valid for complex numbers $u_{i}, v_{i}$ with modulus less than 1 . Therefore, under the different frames of dependence,

\begin{tabular}{c|c|c} 
Dependence & $\left|\operatorname{Cov}\left(F_{t, n}\left(X_{m_{n}}, \ldots, X_{(j-1) m_{n}}\right), G_{t, n}\left(X_{j m_{n}}\right)\right)\right|$ & $T\left(k_{n}\right)$ \\
\hline$\theta$ & $\leq\|t\| \cdot k_{n}^{-1 / 2} \theta_{m_{n}}$ & $\leq\|t\| \cdot k_{n}^{1 / 2} \theta_{m_{n}}$ \\
$\lambda$ & $\leq\|t\| \cdot k_{n}^{1 / 2} \lambda_{m_{n}}$ & $\leq\|t\| \cdot k_{n}^{3 / 2} \lambda_{m_{n}}$
\end{tabular}

and then, Theorem 1 implies Proposition 4.1. 
Proof of Proposition 4.2. Let $x \in \mathbb{R}$, define,

$$
\begin{aligned}
S_{n}=\sqrt{n h_{n}}\left(\widehat{f}_{X}^{(n)}(x)-\mathbb{E} \widehat{f}_{X}^{(n)}(x)\right)=\sum_{i=1}^{n} Y_{i} \\
\quad \text { where } \quad Y_{i}=\frac{1}{\sqrt{n h_{n}}}\left(K\left(\frac{x-X_{i}}{h_{n}}\right)-\mathbb{E}\left(K\left(\frac{x-X_{i}}{h_{n}}\right)\right)\right)=u\left(X_{i}\right)
\end{aligned}
$$

and the function $u$ depends also on $x$ and $n$. First, for $\delta>0$,

$$
\begin{aligned}
A_{n} & =\sum_{i=1}^{n} \mathbb{E}\left(\left\|Y_{i}\right\|^{2+\delta}\right) \\
& =\left(n h_{n}\right)^{-\delta / 2} \cdot \frac{1}{h_{n}} \mathbb{E}\left(\left|K\left(\frac{x-X_{i}}{h_{n}}\right)-\mathbb{E}\left(K\left(\frac{x-X_{i}}{h_{n}}\right)\right)\right|^{2+\delta}\right) \\
& \leq 2^{2+\delta} \cdot\left(n h_{n}\right)^{-\delta / 2} \cdot \frac{1}{h_{n}} \mathbb{E}\left(\left|K\left(\frac{x-X_{i}}{h_{n}}\right)\right|^{2+\delta}\right) \\
& \leq 2^{2+\delta} \cdot\left(n h_{n}\right)^{-\delta / 2} \cdot\left\|f_{X}\right\|_{\infty} \cdot \int_{\mathbb{R}}|K(s)|^{2+\delta} \mathrm{d} s,
\end{aligned}
$$

(the boundedness of $K$ implies the convergence of the last integral). As a consequence, $A_{n} \underset{n \rightarrow \infty}{\longrightarrow} 0$ when $n h_{n} \underset{n \rightarrow \infty}{\longrightarrow} \infty$. Now,

$$
\begin{aligned}
\left|\mathbb{E}\left(Y_{i}\right)\right| \leq \mathbb{E}\left|Y_{i}\right| & \leq\left\|f_{X}\right\|_{\infty} \int_{\mathbb{R}}|u(s)| \mathrm{d} s \\
& \leq \frac{2 h_{n}}{\sqrt{n h_{n}}}\left\|f_{X}\right\|_{\infty} \int|K(v)| \mathrm{d} v \\
& \leq C_{1} \sqrt{\frac{h_{n}}{n}}, \quad \text { for some constant } C_{1}>0 .
\end{aligned}
$$

Moreover, using changes in variables $v=(x-s) / h_{n}, v^{\prime}=\left(x-s^{\prime}\right) / h_{n}$,

$$
\begin{aligned}
\operatorname{Cov}\left(Y_{j}, Y_{i}\right) & =\int_{\mathbb{R}^{2}} u(s) u\left(s^{\prime}\right)\left(f_{j, i}\left(s, s^{\prime}\right)-f_{X}(s) f_{X}\left(s^{\prime}\right)\right) \mathrm{d} s \mathrm{~d} s^{\prime} \\
\left|\operatorname{Cov}\left(Y_{j}, Y_{i}\right)\right| & \leq\left(\left\|f_{j, i}\right\|_{\infty}+\left\|f_{X}\right\|_{\infty}^{2}\right) \int_{\mathbb{R}^{2}}\left|u(s) \| u\left(s^{\prime}\right)\right| \mathrm{d} s \mathrm{~d} s^{\prime} \\
& \leq \frac{4 h_{n}^{2}}{n h_{n}}\left(\left\|f_{j, i}\right\|_{\infty}+\left\|f_{X}\right\|_{\infty}^{2}\right)\left(\int|K(v)| \mathrm{d} v\right)^{2} \\
& \leq C_{2} \frac{h_{n}}{n}, \quad \text { for some constant } C_{2}>0 .
\end{aligned}
$$

The function $K$ is supposed to be a bounded and Lipschitz function, the same for $u$,

$$
\|u\|_{\infty} \leq 2\|K\|_{\infty} \cdot \frac{1}{\sqrt{n h_{n}}} \text { and } \operatorname{Lip} u=2 \operatorname{Lip} K \cdot \frac{1}{h_{n} \sqrt{n h_{n}}}
$$


Therefore, using the $\eta$-weak dependence inequality of the time series $\left(X_{i}\right)_{i \in \mathbb{N}}$, with always $Y_{i}=u\left(X_{i}\right)$, there exists $C>0$ such that,

$$
\left|\operatorname{Cov}\left(Y_{0}, Y_{r}\right)\right| \leq C \cdot u_{n, r} \text { with } u_{n, r}=\left\{\begin{array}{cl}
\frac{\theta_{r}}{n h_{n}^{2}} & \text { for the } \theta \text {-weak dependence } \\
\frac{\lambda_{r}}{n h_{n}^{3}} & \text { for the } \lambda \text {-weak dependence. }
\end{array}\right.
$$

As a consequence of both (12) and the previous inequalities, there exists constants $C_{3}>0$ such that

$$
\left|\operatorname{Cov}\left(Y_{0}, Y_{r}\right)\right| \leq C_{3} \cdot\left(\frac{h_{n}}{n} \wedge u_{n, r}\right), \quad \text { for all } r \in \mathbb{N} .
$$

Finally, we also quote that for $i \in \mathbb{N}$,

$$
\begin{aligned}
\operatorname{Var}\left(Y_{i}\right) & =\int_{\mathbb{R}} f_{X}(t) \cdot u^{2}(t) \mathrm{d} t \\
& =\frac{1}{n} \int_{\mathbb{R}} f_{X}\left(x-h_{n} s\right) \cdot K^{2}(s) \mathrm{d} s-\frac{h_{n}}{n}\left(\int_{\mathbb{R}} f_{X}\left(x-h_{n} s\right) \cdot K(s) \mathrm{d} s\right)^{2} .
\end{aligned}
$$

From the assumptions on functions $f_{X}$ and $K$, the Lebesgue dominated convergence Theorem can be applied and therefore,

$$
n \cdot \operatorname{Var}\left(Y_{i}\right) \underset{n \rightarrow \infty}{\longrightarrow} \int_{\mathbb{R}} f_{X}(x) \cdot K^{2}(s) \mathrm{d} s .
$$

Using the relations (11), (13) and (14), then,

$$
\begin{aligned}
\operatorname{Var}\left(S_{n}\right) & =n \cdot \operatorname{Var} Y_{0}+2 \sum_{i=1}^{n-1}(n-i) \operatorname{Cov}\left(Y_{0}, Y_{i}\right) \\
\left|n h_{n} \cdot \operatorname{Var}\left(\widehat{f}_{X}^{(n)}(x)\right)-f_{X}(x) \int_{\mathbb{R}} K^{2}(t) \mathrm{d} t\right| & \leq o(1)+2 C_{3} \cdot \sum_{i=1}^{n-1}\left(h_{n} \wedge\left(n \cdot u_{n, r i}\right)\right) .
\end{aligned}
$$

Under the assumptions of the Proposition, if the right term of the forthcoming inequality (16) converges to 0 , the right term of the previous inequality converges to 0 and thus,

$$
n h_{n} \cdot \operatorname{Var}\left(\widehat{f}_{X}^{(n)}(x)\right) \underset{n \rightarrow \infty}{\longrightarrow} f(x) \int_{\mathbb{R}} K^{2}(t) \mathrm{d} t .
$$

Now, we are going to bound $T(n)$ for applying Lemma 3 . Let $x \in \mathbb{R}$ and $t \in \mathbb{R}$. First we can write,

$$
T(n)=\sum_{j=1}^{n-1}\left|\operatorname{Cov}\left(F_{x, t}\left(X_{1}, \ldots, X_{j-1}\right), G_{x, t}\left(X_{j}\right)\right)\right|,
$$

where $F_{x, t}\left(X_{1}, \ldots, X_{j-1}\right)=\exp \left(i t\left(Y_{1}+\cdots+Y_{j-1}\right)\right)$ and $G_{x, t}\left(X_{j}\right)=\exp \left(i t Y_{j}\right)$, with always $Y_{k}=u\left(X_{k}\right)$. In order to compute a bound for $T(n)$ we need the following decomposition due to Rio [18],

$$
F_{x, t}\left(X_{1}, \ldots, X_{j-1}\right)=\sum_{k=1}^{j-1}\left(\mathrm{e}^{i t S_{k}}-\mathrm{e}^{i t S_{k-1}}\right), \quad \text { with } S_{k}=Y_{1}+\cdots+Y_{k} \text { and } S_{0}=0
$$


Thus,

$$
\operatorname{Cov}\left(F_{x, t}\left(X_{1}, \ldots, X_{j-1}\right), G_{x, t}\left(X_{j}\right)\right)=\sum_{k=1}^{j-1} \operatorname{Cov}\left(\mathrm{e}^{i t S_{k}}-\mathrm{e}^{i t S_{k-1}}, \mathrm{e}^{i t Y_{j}}\right) .
$$

Consider a r.v. $Y_{j}^{*}$ independent from $\left(Y_{1}, \ldots, Y_{k-1}\right)$, with the same distribution than $Y_{j}$. Then,

$$
\begin{aligned}
\left|\operatorname{Cov}\left(\mathrm{e}^{i t S_{k}}-\mathrm{e}^{i t S_{k-1}}, \mathrm{e}^{i t Y_{j}}\right)\right| & =\left|\mathbb{E}\left(\left(\mathrm{e}^{i t S_{k}}-\mathrm{e}^{i t S_{k-1}}\right)\left(\mathrm{e}^{i t Y_{j}}-\mathrm{e}^{i t Y_{j}^{*}}\right)\right)\right| \\
& \leq|t|^{2} \mathbb{E}\left(\left|Y_{k}\right| \cdot\left(\left|Y_{j}\right|+\left|Y_{j}^{*}\right|\right)\right) \text { from inequality }\left|\mathrm{e}^{i a}-\mathrm{e}^{i b}\right| \leq|b-a| \\
& \leq C \frac{h_{n}}{n}, \quad \text { for some constant } C>0,
\end{aligned}
$$

from relations (11) and (12). From another hand, one can write,

$$
\operatorname{Cov}\left(\mathrm{e}^{i t S_{k}}-\mathrm{e}^{i t S_{k-1}}, \mathrm{e}^{i t Y_{j}}\right)=\operatorname{Cov}\left(g_{1}\left(X_{1}, \ldots, X_{k}\right), g_{2}\left(X_{j}\right)\right),
$$

with $\left\|g_{2}\right\|_{\infty}=1$ and $\left\|g_{1}\right\|_{\infty} \leq|t|\left\|S_{k}-S_{k-1}\right\|_{\infty} \leq|t| \cdot\|u\|_{\infty} \leq 2|t|\|K\|_{\infty} \cdot \frac{1}{\sqrt{n h_{n}}}$ and,

$$
\begin{aligned}
\frac{\mid \mathrm{e}^{i t\left(u\left(x_{1}\right)+\cdots+u\left(x_{k}\right)\right)}-\mathrm{e}^{i t\left(u\left(y_{1}\right)+\cdots+u\left(y_{k}\right)\right) \mid}}{\left|x_{1}-y_{1}\right|+\cdots+\left|x_{k}-y_{k}\right|} & \leq|t| \cdot \frac{\left|\left(u\left(x_{1}\right)+\cdots+u\left(x_{k}\right)\right)-\left(u\left(y_{1}\right)+\cdots+u\left(y_{k}\right)\right)\right|}{\left|x_{1}-y_{1}\right|+\cdots+\left|x_{k}-y_{k}\right|} \\
& \leq|t| \cdot \operatorname{Lip} u \cdot k .
\end{aligned}
$$

As a consequence, $\operatorname{Lip} g_{1} \leq 8|t| \cdot \operatorname{Lip} K \cdot \frac{k}{h_{n} \sqrt{n h_{n}}} \quad$ and $\operatorname{Lip} g_{2} \leq 4|t| \cdot \operatorname{Lip} K \cdot \frac{1}{h_{n} \sqrt{n h_{n}}}$. Using these results and the weak dependence property of $X$, there exists $C>0$ such that for $\ell=j-k \in[1, j]$,

$$
\begin{aligned}
\left|\operatorname{Cov}\left(\mathrm{e}^{i t S_{k}}-\mathrm{e}^{i t S_{k-1}}, \mathrm{e}^{i t Y_{j}}\right)\right| \leq C \cdot \frac{h_{n}}{n} \wedge v_{n, k, \ell} \\
\text { with } v_{n, k, \ell}= \begin{cases}k^{2}\left(\frac{1}{n h_{n}^{3}} \vee \frac{1}{n^{1 / 2} h_{n}^{3 / 2}}\right) \cdot \lambda_{\ell} & \text { for the } \lambda \text {-weak dependence, } \\
\frac{1}{n h_{n}^{2}} \cdot \theta_{\ell} & \text { for the } \theta \text {-weak dependence. }\end{cases}
\end{aligned}
$$

This implies,

$$
\begin{aligned}
T(n) & \leq C \sum_{j=1}^{n} \sum_{\ell=1}^{j} \frac{h_{n}}{n} \wedge v_{n, n, \ell} \\
& \leq C \sum_{\ell=1}^{n} h_{n} \wedge\left(n \cdot v_{n, n, \ell}\right) \\
& \leq C \cdot h_{n}^{1-\beta} n^{\beta} \sum_{\ell=1}^{n} v_{n, n, \ell}^{\beta}
\end{aligned}
$$

with $\beta \geq 0$ (analogously to the case of $\operatorname{Var} \widehat{f}_{X}^{(n)}(x)$ ). Since $n h_{n} \underset{n \rightarrow \infty}{\longrightarrow} \infty$ and $h_{n} \underset{n \rightarrow \infty}{\longrightarrow} 0$, one obtains that $T(n) \underset{n \rightarrow \infty}{\longrightarrow} 0$ under the different conditions satisfied by $h_{n}$ and the weak dependence sequence. Then, all the conditions of Theorem 1 are satisfied, which implies Proposition 4.2. 
Proof of Corollaries 4.1 and 4.2. Under the assumptions on $K$, from Prakasa Rao [16],

$$
\mathbb{E}\left(\widehat{f}_{X}^{(n)}(x)\right)= \begin{cases}f_{X}(x)+h_{n}^{p} \cdot(1+o(1)) \cdot f_{X}^{(p)}(x) \frac{1}{p !} \int_{\mathbb{R}} t^{p} K(t) \mathrm{d} t & \text { if the regularity of } f_{X} \text { is } p \in \mathbb{N}^{*} \\ f_{X}(x)+O\left(h_{n}^{[\rho]}\right) & \text { if the regularity of } f_{X} \text { is } \rho \notin \mathbb{N}^{*}\end{cases}
$$

It implies the optimal choice of convergence rate of $h_{n}$, following this two cases, and the conditions on the convergence rates of the different frames of weak dependent.

Proof of Proposition 4.3. This proof is quite the same than the proof of Proposition 4.2 and therefore we omit the details. Let $x \in \mathbb{R}$, define,

$$
\begin{aligned}
S_{n}=\sqrt{k_{n} h_{n}}\left(\widehat{f}_{X}^{\left(n, m_{n}\right)}(x)-\mathbb{E} \widehat{f}_{X}^{\left(n, m_{n}\right)}(x)\right) & =\sum_{i=1}^{k_{n}} Y_{i} \\
\text { where } \quad Y_{i} & =\frac{1}{\sqrt{k_{n} h_{n}}}\left(K\left(\frac{x-X_{i m_{n}}}{h_{n}}\right)-\mathbb{E}\left(K\left(\frac{x-X_{i m_{n}}}{h_{n}}\right)\right)\right)=u\left(X_{i m_{n}}\right),
\end{aligned}
$$

and the function $u$ depends both on $x$ and $n$. First, for $\delta>0$,

$$
A_{n}^{\left(m_{n}\right)}=\sum_{i=1}^{k_{n}} \mathbb{E}\left(\left\|Y_{i}\right\|^{2+\delta}\right) \leq 2 \cdot\left(k_{n} h_{n}\right)^{-\delta / 2} \cdot\left\|f_{X}\right\|_{\infty} \cdot \int_{\mathbb{R}}|K(s)|^{2+\delta} \mathrm{d} s .
$$

As a consequence, $A_{n}^{\left(m_{n}\right)} \underset{n \rightarrow \infty}{\longrightarrow} 0$ when $k_{n} h_{n} \underset{n \rightarrow \infty}{\longrightarrow} \infty$. Moreover, we have,

$$
\begin{aligned}
\left|\mathbb{E}\left(Y_{i}\right)\right| & \leq C_{1} \sqrt{\frac{h_{n}}{k_{n}}}, \quad \text { for some constant } C_{1}>0 \\
\left|\operatorname{Cov}\left(Y_{0}, Y_{r}\right)\right| & \leq C_{3} \cdot\left(\frac{h_{n}}{k_{n}} \wedge u_{k_{n}, m_{n} \cdot r}\right), \quad \text { for some constant } C_{3}>0 \text { and all } r \in \mathbb{N},
\end{aligned}
$$

with the sequence $\left(u_{p, q}\right)$ defined in the proof of the Proposition 4.2. We have also,

$$
k_{n} \cdot \operatorname{Var}\left(Y_{i}\right) \underset{n \rightarrow \infty}{\longrightarrow} \int_{\mathbb{R}} f_{X}(x) \cdot K^{2}(s) \mathrm{d} s
$$

and thus $\left|k_{n} h_{n} \cdot \operatorname{Var}\left(\widehat{f}_{X}^{\left(n, m_{n}\right)}(x)\right)-f_{X}(x) \int_{\mathbb{R}} K^{2}(t) d t\right| \leq o(1)+2 C_{3} \cdot \sum_{i=1}^{k_{n}-1}\left(h_{n} \wedge\left(k_{n} \cdot u_{k_{n}, m_{n} \cdot r}\right)\right)$. Under the conditions on $h_{n}$ and $m_{n}$,

$$
k_{n} h_{n} \cdot \operatorname{Var}\left(\widehat{f}_{X}^{\left(n, m_{n}\right)}(x)\right) \underset{n \rightarrow \infty}{\longrightarrow} f(x) \int_{\mathbb{R}} K^{2}(t) \mathrm{d} t .
$$

For bounding $T\left(k_{n}\right)$, one writes again with $S_{k}=Y_{1}+\cdots+Y_{k}$ and $S_{0}=0$,

$$
T\left(k_{n}\right)=\sum_{j=1}^{k_{n}-1}\left|\sum_{k=1}^{j-1} \operatorname{Cov}\left(\mathrm{e}^{i t S_{k}}-\mathrm{e}^{i t S_{k-1}}, \mathrm{e}^{i t Y_{j}}\right)\right| .
$$

Thanks to the inequality,

$$
\left|\operatorname{Cov}\left(\mathrm{e}^{i t S_{k}}-\mathrm{e}^{i t S_{k-1}}, \mathrm{e}^{i t Y_{j}}\right)\right| \leq C \frac{h_{n}}{k_{n}}, \quad \text { for some constant } C>0
$$


and with the sequence $\left(v_{n, k, \ell}\right)$ of the previous proof, this implies,

$$
\begin{aligned}
T\left(k_{n}\right) & \leq C \cdot \sum_{i=1}^{k_{n}}\left(h_{n} \wedge\left(k_{n} \cdot v_{k_{n}, k_{n}, m_{n} \ell}\right)\right) \\
& \leq C \cdot h_{n}^{1-\beta} k_{n}^{\beta} \sum_{\ell=1}^{k_{n}} v_{k_{n}, k_{n}, m_{n} \ell}^{\beta}
\end{aligned}
$$

with $\beta \geq 0$. With $h_{n}=n^{-h}$ and $m_{n}=n^{m}$, where $h, m \in(0,1)$, the condition $T\left(k_{n}\right) \underset{n \rightarrow \infty}{\longrightarrow} 0$, which implies (18) and therefore the central limit theorem, can be obtained for different choice of $h$ and $m$. After computations, the following graphs provide the zonas (depending also of the decay rate $\theta$ or $\lambda$ of weak dependence property) where $h$ and $m$ can be chosen.

Finally, the "optimal" rate of convergence, in the sense of a maximal $\sqrt{k_{n} h_{n}}=n^{(1-m-h) / 2}$, is obtained from a maximization of $1-m-h$. In every cases this occurs for the point $(m, h)$ most below and left of the graph "white" zona. This implies the optimal condition of the proposition.

\section{REFERENCES}

[1] D. Andrews, Non strong mixing autoregressive processes. J. Appl. Probab. 21 (1984) 930-934.

[2] P. Billingsley, Convergence of Probability Measures. Wiley, New-York (1968).

[3] A.V. Bulinski and A.P. Shashkin, Rates in the central limit theorem for weakly dependent random variables. J. Math. Sci. 122 (2004) 3343-3358.

[4] A.V. Bulinski and A.P. Shashkin, Strong Invariance Principle for Dependent Multi-indexed Random Variables. Doklady Mathematics 72 (2005) 503-506.

[5] C. Coulon-Prieur and P. Doukhan, A triangular central limit theorem under a new weak dependence condition. Stat. Prob. Letters 47 (2000) 61-68.

[6] P. Doukhan, Mixing: Properties and Examples. Lect. Notes Statis. 85 (1994).

[7] P. Doukhan, Models inequalities and limit theorems for stationary sequences, in Theory and applications of long range dependence, Doukhan et al. Ed., Birkhäuser (2003) 43-101.

[8] P. Doukhan and G. Lang, Rates in the empirical central limit theorem for stationary weakly dependent random fields. Stat. Inference Stoch. Process. 5 (2002) 199-228.

[9] P. Doukhan and S. Louhichi, A new weak dependence condition and applications to moment inequalities. Stoch. Proc. Appl. 84 (1999) 313-342.

[10] P. Doukhan, H. Madre and M. Rosenbaum, Weak dependence for infinite ARCH-type bilinear models. Statistics 41 (2007) $31-45$.

[11] P. Doukhan, G. Teyssiere and P. Winant, Vector valued $\mathrm{ARCH}(\infty)$ processes, in Dependence in Probability and Statistics, P. Bertail, P. Doukhan and P. Soulier Eds. Lecture Notes in Statistics, Springer, New York (2006).

[12] P. Doukhan and O. Wintenberger, An invariance principle for weakly dependent stationary general models. Prob. Math. Stat. 27 (2007) 45-73.

[13] L. Giraitis and D. Surgailis, ARCH-type bilinear models with double long memory. Stoch. Proc. Appl. 100 (2002) 275-300.

[14] M.H. Neumann and E. Paparoditis, Goodness-of-fit tests for Markovian time series models. Technical Report No. 16/2005. Department of Mathematics and Statistics, University of Cyprus (2005).

[15] V. Petrov, Limit theorems of probability theory. Clarendon Press, Oxford (1995).

[16] B.L.S. Prakasha Rao, Nonparametric functional estimation. Academic Press, New York (1983).

[17] E. Rio, About the Lindeberg method for strongly mixing sequences. ESAIM: PS 1 (1997) 35-61.

[18] E. Rio, Théorie asymptotique pour des processus aléatoires faiblement dépendants. SMAI, Math. Appl. 31 (2000).

[19] P.M. Robinson, Nonparametric estimators for time series. J. Time Ser. Anal. 4 (1983) 185-207.

[20] M.S. Taqqu, Weak convergence to fractional Brownian motion and to the Rosenblatt process. Z. Wahrsch. Verw. Gebiete 31 (1975) 237-302. 